\title{
(Un)countable and (non)effective versions of Ramsey's theorem
}

\author{
Dietrich Kuske
}

\begin{abstract}
We review Ramsey's theorem and its extensions by Jockusch for computable partitions, by Sierpiński and by Erdős and Rado for uncountable homogeneous sets, by Rubin for automatic partitions, and by the author for $\omega$-automatic (in particular uncountable) partitions.
\end{abstract}

\section{Introduction}

Every infinite graph has an infinite clique or an infinite anticlique - this is the paradigmatic formulation of Ramsey's theorem [14]. But this theorem is highly non-constructive since there are computable infinite graphs none of its infinite cliques and anticliques is computable (they are not even in $\Sigma_{2}^{0}[\mathbf{7}]$ ). On the positive side, Jockusch also showed that every infinite computable graph contains an infinite clique or anticlique from $\Pi_{2}^{0}$. Soon after Ramsey's paper from 1930, authors got interested in a quantitative analysis: how many nodes are necessary and sufficient to obtain a clique or anticlique of size $\aleph_{1}$. The answer was given by Sierpiński $[\mathbf{1 7}]$ $\left(\left(2^{\aleph_{0}}\right)^{+}\right.$nodes are necessary) and Erdős \& Rado [6] $\left(\left(2^{\aleph_{0}}\right)^{+}\right.$nodes are sufficient $)$. We unify the proofs of these positive results by the notion of a Ramsey tree. In doing so, we prove in particular that every infinite computable finitely branching tree contains an infinite path from $\Pi_{2}^{0}$.

Recall that a graph is computable if both its set of nodes and its set of edges can be decided by a Turing machine. Replacing these Turing machines by finite automata, one obtains the more restrictive notion of an automatic graph: the set of nodes is a regular set and whether a pair of nodes forms an edge can be decided by a synchronous two-tape automaton (this concept is known since the beginning of automata theory, a systematic study started with $[\mathbf{9}, \mathbf{3}]$, see $[\mathbf{1 5}]$ for a recent overview). Compared to computable graphs, here, the situation is much more favourable: every infinite automatic graph contains an infinite regular clique or an infinite regular anticlique [15].

Since automatic graphs contain at most $\aleph_{0}$ nodes, we need a more general notion for a recursion-theoretic analysis of uncountable graphs. For this, we use

2010 Mathematics Subject Classification. Primary 03D05, 05 D10.

Key words and phrases. Computable and automatic graphs, uncountable graphs, Ramsey theory.

(C)0000 (copyright holder) 
Blumensath \& Grädel's [3] $\omega$-automatic graphs: the names of nodes form a regular $\omega$-language and the edge relation (on names) as well as the relation "these two names denote the same node" can be decided by a synchronous 2-tape Büchi-automaton. We show that any such graph of size $2^{\aleph_{0}}$ has a clique or anticlique of size $2^{\aleph_{0}}$, but these (anti)cliques are not necessarily described by a Büchi-automaton.

While the results by Ramsey, by Jockusch, by Erdős \& Rado, and by Rubin generalise to hypergraphs, this is not the case for our result on $\omega$-automatic graphs: We present a ternary $\omega$-automatic hypergraph of size $2^{\aleph_{0}}$ that does not contain any uncountable clique or anticlique.

Finally, it should be noted that all the positive results, except those by Rubin and by Kuske are shown by an analysis of the Ramsey tree of a hypergraph. This proof technique seems not to work in the $(\omega$-)autmatic setting where other strategies are employed.

Acknowledgment Markus Lohrey's careful and sympathetic reading of the manuscript eliminated more mistakes than I thought possible. I am very grateful for the time and energy he devoted to this task.

\section{Partitions and the Ramsey tree}

For a set $V$ and a natural number $k \geq 1$, let $[V]^{k}$ denote the set of $k$-element subsets of $V$. A $(k, \ell)$-partition is a tuple $P=\left(V, E_{1}, \ldots, E_{\ell}\right)$ where $V$ is a set and $\left(E_{1}, \ldots, E_{\ell}\right)$ is a partition of $[V]^{k}$ into (possibly empty) sets. For $1 \leq i \leq \ell$, a set $W \subseteq V$ is $E_{i}$-homogeneous if $[W]^{k} \subseteq E_{i}$; it is homogeneous if it is $E_{i^{-}}$ homogeneous for some $1 \leq i \leq \ell$. The case $k=\ell=2$ is special: any $(2,2)$-partition $G=\left(V, E_{1}, E_{2}\right)$ can be considered as an (undirected loop-free) graph $\left(V, E_{1}\right)$. Homogeneous sets in $G$ are then complete or discrete induced subgraphs of $\left(V, E_{1}\right)$.

This paper is concerned with the following question: Does every $(k, \ell)$-partition $G=\left(V, E_{1}, \ldots, E_{\ell}\right)$ with $|V|=\kappa$ have a homogeneous set of size $\lambda$ (where $\kappa$ and $\lambda$ are cardinal numbers and $k, \ell \geq 2$ are natural numbers). If this is the case, one writes

$$
\kappa \rightarrow(\lambda)_{\ell}^{k}
$$

(a notation due to Erdős \& Rado [5]).

OBservation 1.1. Let $P=\left(V, E_{1}, \ldots, E_{\ell}\right)$ be a $(k, \ell)$-partition, $\leq$ a well-order on $V$, and $v \in V$. Then there exists a unique ordinal sequence $\left(v_{\alpha}\right)_{\alpha \leq \beta}$ satisfying: (R1) for $\alpha \leq \beta$, the node $v_{\alpha}$ is the least element of $(V, \leq)$ such that

- $v_{\gamma}<v_{\alpha}$ for all $\gamma<\alpha$ and

- $A \cup\left\{v_{\alpha}\right\} \in E_{i}$ iff $A \cup\{v\} \in E_{i}$ for all $A \in\left[\left\{v_{\gamma} \mid \gamma<\alpha\right\}\right]^{k-1}$ and $1 \leq i \leq \ell$

(R2) $v_{\beta}=v$

EXAMPLE 1.2. We consider the Rado graph as a $(2,2)$-partition $\left(\mathbb{N}, E_{1}, E_{2}\right)$ where $\{m, n\} \in E_{1}$ with $m<n$ if, in the binary expansion of $n$, position $m$ carries 1 (i.e., the unique presentation of $n$ as sum of powers of 2 contains the summand $2^{m}$ ).

For a word $w$ over $\{0,1\}$, let $[w]$ denote its value at base 2 , i.e., $[\varepsilon]=0$ and $[w x]=2 \cdot w+x$ for $x \in\{0,1\}$.

Then $0,1,5,33,37$ is the Ramsey sequence of 37: Clearly, every Ramsey sequence has to start with $v_{0}=0$. Then $[100101]=37$, so $\{0,37\} \in E_{1}$. Hence we determine the minimal number $v_{1}>v_{0}$ whose binary expansion ends with 1 - this is obviously $v_{1}=1$. Since $\{1,37\} \in E_{2}$, we now search for the minimal number 
$v_{2}>v_{1}=1$ whose binary expansion ends in $01-$ hence $v_{2}=[101]=5$. Again, $\{5,37\} \in E_{1}$, so $v_{3}$ is the minimal number above 5 whose binary expansion ends with $1 a b c 01$ for some $a, b, c \in\{0,1\}$ - hence $v_{3}=[100001]=33$. Now $\{33,37\} \in E_{2}$, so $v_{4}$ is the minimal number whose binary expansion ends with $0 w 1 a b c 01$ with $w \in\{0,1\}^{*}$ of length 28 and $a, b, c \in\{0,1\}-$ thus $v_{4}=[100101]=37$.

Let $v \in V$. Then one can construct, by transfinite induction, the sequence of nodes $v_{\alpha}$ satisfying (R1). In particular, $v_{0}<v_{1}<\cdots<v_{k-2}$ is the initial segment of $(V, \leq)$ of length $k-1$ (if $v$ is not among these elements). Eventually, $v$ is the least element satisfying the two conditions in (R1) - at this stage $\beta$, the process terminates. We call the sequence $\left(v_{\alpha}\right)_{\alpha \leq \beta}$ the Ramsey sequence of $v$ and denote it by $\bar{v}$; its length is $\beta$. Using these sequences, we can define a partial order $\preceq$ on $V$ by setting $v \preceq w$ if and only if $\bar{v}$ is a prefix of $\bar{w}$ (which is the case if and only if $v$ appears in the sequence $\bar{w})$. The structure $(V, \preceq)$ is the Ramsey tree of the $(k, \ell)$-partition $P$ with respect to the well-order $\leq$.

Some properties of the Ramsey tree are obvious:

(1) If $v \in V$, then its predecessors in the Ramsey tree (i.e., the set $\{w \in V \mid$ $w \preceq v\})$ are well-ordered. This justifies to call $(V, \preceq)$ an order tree.

(2) Let $v \in V$ and let $B$ be the cardinality of its Ramsey sequence. For any two distinct brothers $x$ and $y$ of $v$, there exists a $(k-1)$-elements subset $A$ of $\bar{v}$ not containing $v$ and $1 \leq i \leq \ell$ such that $A \cup\{x\} \in E_{i}$ and $A \cup\{y\} \notin E_{i}$. Hence the number of brothers is at most

$$
\ell^{\left(\begin{array}{c}
B \\
k-1
\end{array}\right)}=\ell^{\left|[B]^{k-1}\right|} .
$$

In particular, it is finite if $\bar{v}$ is finite, and it is at most $2^{\aleph_{n}}$ if $|B|=\aleph_{n}$.

Different versions of Ramsey's theorem will be derived from an analysis of the Ramsey tree. This is based on the following observation and result that allows to infer the existence of homogeneous sets in $(k, \ell)$-partitions from their existence in $(k-1, \ell)$-partitions.

ObSERVATIOn 1.3. Let $(V, \preceq)$ be the Ramsey tree of the $(k, \ell)$-partition $P=$ $\left(V, E_{1}, \ldots, E_{\ell}\right)$ with respect to the well-order $\leq$ and let $Y \subseteq V$ be some chain in the Ramsey tree (i.e., linearly ordered subset) without maximal element.

For $A \in[Y]^{k-1}$, set $A \in E_{i}^{Y}$ if and only if there exists $y \in Y$ with $A \prec y$ and $A \cup\{y\} \in E_{i}$. Then $P^{Y}=\left(Y, E_{1}^{Y}, \ldots, E_{\ell}^{Y}\right)$ is a $(k-1, \ell)$-partition.

Proof. Since $Y$ has no maximal element with respect to $\preceq$, any set $A \in[Y]^{k-1}$ belongs to some $E_{i}^{Y}$. Suppose $A \in E_{i}^{Y} \cap E_{j}^{Y}$. Then there are $y_{i}, y_{j} \in Y$ with $A \prec y_{i}, y_{j}, A \cup\left\{y_{i}\right\} \in E_{i}$, and $A \cup\left\{y_{j}\right\} \in E_{j}$. For symmetry reasons, we can assume $y_{i} \preceq y_{j}$. But then $i=j$ by (R1) since $y_{i}$ appears in the Ramsey sequence of $y_{j}$.

Proposition 1.4. Let $k \geq 2$ and let $P=\left(V, E_{1}, \ldots, E_{\ell}\right)$ be $a(k, \ell)$-partition and $Y$ a chain without maximal element in the Ramsey tree $(V, \preceq)$. Any set homogeneous in the $(k-1, \ell)$-partition $P^{Y}$ induced by $Y$ is homogeneous in $P$.

Proof. Let $W \subseteq Y \subseteq V$ be $E_{i}^{Y}$-homogeneous, i.e., $[W]^{k-1} \subseteq E_{i}^{Y}$. Let $B \in$ $[W]^{k} \subseteq[Y]^{k}$ and let $v<w$ be the maximal elements of $(B, \leq)$. Then $A:=B \backslash\{w\} \in$ $[W]^{k-1} \subseteq E_{i}^{Y}$. By the definition of $E_{i}^{Y}$, there exists $y \in Y$ with $A \leq v<y$ and $A \cup\{y\} \in E_{i}$. Since $(Y, \preceq)$ is linearly ordered, $y$ appears in the Ramsey sequence of $w$ or vice versa. In any case, $B=A \cup\{w\} \in E_{i}$ follows from (R1). 


\section{The countable case}

\subsection{Ramsey's theorem.}

TheOREM 2.1 (Ramsey [14]). If $k, \ell \geq 1$ and $\kappa \geq \aleph_{0}$, then $\kappa \rightarrow\left(\aleph_{0}\right)_{\ell}^{k}$.

PROOF. It suffices to prove the theorem for $\kappa=\aleph_{0}$ in which case it is shown by induction on $k$. The base case $(k=1)$ is the pigeonhole principle. So suppose $\aleph_{0} \rightarrow\left(\aleph_{0}\right)_{\ell}^{k-1}$ and let $P=\left(\mathbb{N}, E_{1}, \ldots, E_{\ell}\right)$ be a $(k, \ell)$-partition. The well-order $\leq$ on the base set $\mathbb{N}$ of this partition is the natural order on the natural numbers. Then the Ramsey tree of $P$ is infinite and, since all Ramsey sequences are finite, finitely branching. Hence, by König's lemma, it contains some infinite branch $Y$ (that does not have a maximal element). Then $P^{Y}$ is an infinite $(k-1, \ell)$-partition, so it contains by the induction hypothesis an infinite homogeneous set. But this set is, by Prop. 1.4 also homogeneous in $P$.

2.2. Jockusch's theorems. A partition $\left(\mathbb{N}, E_{1}, \ldots, E_{\ell}\right)$ is computable if all the sets $E_{i}$ are decidable. For $k, \ell \geq 2$ and classes of sets $\mathcal{C} \subseteq 2^{[\mathbb{N}]^{k}}$ and $\mathcal{D} \subseteq 2^{\mathbb{N}}$, write

$$
\left(\aleph_{0}, \mathcal{C}\right) \rightarrow\left(\aleph_{0}, \mathcal{D}\right)_{\ell}^{k}
$$

if every $(k, \ell)$-partition $\left(\mathbb{N}, E_{1}, \ldots, E_{\ell}\right)$ with $E_{1}, \ldots, E_{\ell} \in \mathcal{C}$ contains some infinite homogeneous set $H \in \mathcal{D}$. In this section, the sets $\mathcal{C}$ and $\mathcal{D}$ will be classes from the arithmetical hierarchy: A set $A \subseteq \mathbb{N}$ belongs to $\Sigma_{n}^{0}$ if and only if there is a computable predicate $P \subseteq \mathbb{N}^{n+1}$ such that

$$
A=\left\{x \in \mathbb{N} \mid \exists x_{1} \forall x_{2} \ldots \exists / \forall x_{n}:\left(x, x_{1}, \ldots, x_{n}\right) \in P\right\} .
$$

The set $A$ belongs to $\Pi_{n}^{0}$ if its complement belongs to $\Sigma_{n}^{0}$, it belongs to $\Delta_{n}^{0}$ if it is both, $\Sigma_{n}^{0}$ and $\Pi_{n}^{0}$, and it belongs to the arithmetical hierarchy $\mathrm{AH}$ if it belongs to $\Sigma_{n}^{0}$ for some $n \in \mathbb{N}$. We also write REC for $\Delta_{1}^{0}$ since these are precisely the computable sets. Identifying a finite set $A \in[\mathbb{N}]^{k}$ with its Gödel number, we can also say that a set $E \subseteq[\mathbb{N}]^{k}$ belongs to $\Sigma_{n}^{0}$ etc.

We now discuss the computational content of the above proof of Ramsey's theorem. First note that clearly

$$
\left(\aleph_{0}, \mathrm{REC}\right) \rightarrow\left(\aleph_{0}, \mathrm{REC}\right)_{\ell}^{1}
$$

since, given an infinite computable $(1, \ell)$-partition, one of the classes $E_{1}, \ldots, E_{\ell}$ is infinite and they are all computable. Next, let $\left(\mathbb{N}, E_{1}, \ldots, E_{\ell}\right)$ be some computable $(k, \ell)$-partition and let $\leq$ be the natural order on $\mathbb{N}$. Then the Ramsey tree of $P$ wrt. $\leq$ has a computable copy: it consists of all Ramsey sequences (that are necessarily finite) ordered by the prefix order. An isomorphism is given by mapping $v$ to its Ramsey sequence $\bar{v}$. In order to apply Prop. 1.4 , we have to find an infinite branch in this computable copy. First note that also the successor relation in this computable copy is computable. We therefore now make a short excursion into the theory of computable successor trees.

Theorem 2.2. Let $T=(V$, succ) with $V \subseteq \mathbb{N}$ be an infinite computable finitely branching successor tree. Then $T$ contains an infinite branch from $\Pi_{2}^{0}$.

Proof. On $V$, we define the lexicographic order: $m \leq_{\text {lex }} n$ if and only if $(m, n) \in$ succ $^{*}$ or there exist nodes $x, m^{\prime}, n^{\prime} \in V$ with $\left(x, m^{\prime}\right),\left(x, n^{\prime}\right) \in$ succ, $\left(m^{\prime}, m\right),\left(n^{\prime}, n\right) \in$ succ $^{*}$, and $m^{\prime}<n^{\prime}$. Note that the linear order $\leq_{\text {lex }}$ is decidable. Furthermore, the root of $T$ is its minimal element and every node $v \in V$ has 
a successor in the linear order $\left(V, \leq_{\text {lex }}\right.$ ) (if it is not maximal in this linear order). Hence $\left(V, \leq_{\text {lex }}\right)$ is isomorphic to $\omega+\left(V^{\prime}, \leq_{\text {lex }}\right)$ for some set $V^{\prime} \subseteq V$. For $n \geq 1$ let $v_{n}$ denote the $n^{\text {th }}$ element of $\left(V, \leq_{\text {lex }}\right)$ and define

$$
C=\left\{v \in V \mid \forall n:\left(v \leq_{\operatorname{lex}} v_{n} \Rightarrow\left(v, v_{n}\right) \in \operatorname{succ}^{*}\right) \wedge \exists m: v=v_{m}\right\} .
$$

This set is a chain in the tree $\left(V\right.$, succ $\left.{ }^{*}\right)$ : Let $v, w \in C$. Then there exist $m, n \in \mathbb{N}$ with $v=v_{m}$ and $w=v_{n}$, we assume $m \leq n$. By the definition, this implies $v_{m} \leq_{\text {lex }}$ $v_{n}$ and therefore (since $v=v_{m} \in C$ ) also $\left(v_{m}, v_{n}\right) \in \operatorname{succ}^{*}$, i.e., $(v, w) \in$ succ $^{*}$.

Our next aim is to show that $C$ is infinite. First note that the root $v_{1}$ belongs to $C$. Let $c \in C$. Then there are infinitely many $m \in \mathbb{N}$ with $c \leq_{\text {lex }} v_{m}$ and therefore with $\left(c, v_{m}\right) \in$ succ* $^{*}$. Let $c^{\prime}$ be the lexicographically largest son of $c$ of the form $v_{n}$. Then, for all $s \geq n$, we have $c<_{\text {lex }} c^{\prime}=v_{n} \leq_{\text {lex }} v_{s}$ implying $\left(c, v_{s}\right) \in$ succ $^{*}$ and therefore $\left(c^{\prime}, v_{s}\right) \in$ succ* $^{*}$. Hence $c^{\prime} \in C$, i.e., the chain $C$ does not contain a maximal element. It follows that $C$ is even an infinite branch (i.e., downwards closed).

To show that $C$ is $\Pi_{2}^{0}$, it suffices to argue that the relation $R=\left\{\left(n, v_{n}\right) \mid n \in \mathbb{N}\right\}$ is $\Sigma_{2}^{0}$. But this is obvious since it is defined by

$$
\begin{aligned}
\exists v_{1}, v_{2}, \ldots, v_{n-1} \forall v: & \bigwedge_{1 \leq i<n-1} v_{i}<_{\text {lex }} v_{i+1} \\
& \wedge \quad v \Varangle_{\operatorname{lex}} v_{1} \wedge \bigwedge_{1 \leq i<n-1} \neg\left(v_{i}<_{\text {lex }} v<_{\text {lex }} v_{i+1}\right) .
\end{aligned}
$$

LEMmA 2.3. Let $P=\left(\mathbb{N}, E_{1}, \ldots, E_{\ell}\right)$ be a computable and infinite $(k+1, \ell)$ partition and let $C \subseteq \mathbb{N}$ be an infinite branch in its Ramsey tree from $\Pi_{2}^{0}$. Then $E_{i}^{C} \subseteq \mathbb{N}$ is $\Pi_{2}^{0}$ for all $1 \leq i \leq \ell$.

Proof. Let $A \in[C]^{k}$ with maximal element $a$. Let furthermore $\mathbb{A}$ denote the finite set $\{x \in V \mid x \preceq a\}$. Then $A \in E_{i}^{C}$ if and only if there exists a function $f:[\mathbb{A}]^{k} \rightarrow\{1, \ldots, \ell\}$ with $f(A)=i$ such that

$$
\begin{aligned}
\forall y(a \prec y \wedge & \left.\bigwedge_{B \in[\mathbb{A}]^{k}} B \cup\{y\} \in E_{f(B)} \rightarrow y \in C\right) \\
\wedge \exists y(x \prec y \wedge & \left.\bigwedge_{B \in[\mathbb{A}]^{k}} B \cup\{y\} \in E_{f(B)}\right)
\end{aligned}
$$

where $\prec$ is the immediate successor relation of the tree $T$. The existentially quantified formula and the premise in the universally quantified formula are computable predicates. Since the quantification over the functions $f$ is computably bounded, the whole expression belongs to $\Pi_{2}^{0}$.

ThEOREM 2.4. Let $P=\left(V, E_{1}, \ldots, E_{\ell}\right)$ be some infinite computable $(2, \ell)$-partition. Then $P$ contains an infinite homogeneous set from $\Pi_{2}^{0}$.

Proof. Since also the successor relation of the Ramsey tree of $P$ is computable, we can apply Theorem 2.2, i.e., there exists an infinite chain $C$ from $\Pi_{2}^{0}$ in the Ramsey tree of $P$. Since $C$ is infinite, one of the sets $E_{i}^{C}$ is infinite. By Lemma 2.3, this set $E_{i}^{C}$ belongs to $\Pi_{2}^{0}$ and, by Prop. 1.4, it is homogeneous in $P$.

Theorem 2.5 (attributed to Manaster in $[7$, p. 276]). For all $k, \ell \geq 1$, we have

$$
\left(\aleph_{0}, \mathrm{AH}\right) \rightarrow\left(\aleph_{0}, \mathrm{AH}\right)_{\ell}^{k}
$$


Proof. Note that Theorem 2.2 and Lemma 2.3 and therefore Theorem 2.4 also hold in their relativized form. Hence we can prove this theorem by induction in the same way that we proved Ramsey's theorem.

Jockusch [7] proved the more precise result

$$
\left(\aleph_{0}, \Delta_{n}^{0}\right) \rightarrow\left(\aleph_{0}, \Pi_{n+k-1}^{0}\right)_{\ell}^{k}
$$

for $k, \ell, n \geq 1$. In general, the proof proceeds by induction as before: from a $(k, \ell)$-partition $P$ whose partition classes all belong to $\Delta_{n}^{0}$, one builds a $(k-1, \ell)$ partition whose homogeneous sets are also homogeneous in $P$. Once the inductive construction reaches a $(2, \ell)$-partition, the above Theorem 2.4 is invoked. While Manaster uses the $\Pi_{2}^{0}$-branch from Theorem 2.2 in the inductive step, Jockusch uses a branch $C$ such that $C^{\prime}$ (the first jump of $C$ ) is $\Delta_{3}^{0}$ - existence of such a branch is shown in $[8]$.

Esthetically, this result is not as satisfactory as the previous ones since the levels of the arithmetical hierarchy on the left and on the right do not coincide. Jockusch [7] also showed that this cannot be avoided. His construction uses the following limit lemma twice.

Lemma 2.6 (Shoenfield [16]). Let $k \geq 2$. There is a total function $F: \mathbb{N}^{3} \rightarrow$ $\{0,1\}$ computable in $\emptyset^{(k-2)}$ such that, for any $A \in \Delta_{k}^{0}$, there is $e \in \mathbb{N}$ satisfying

(1) the sequence $(F(e, m, s))_{s \in \omega}$ is ultimately constant for all $m \in \mathbb{N}$, and

(2) $A=\{m \mid F(e, m, s)=1$ for almost all $s\}$.

Proof. For $s \in \mathbb{N}$ and $X \subseteq \mathbb{N}$, let $K_{s}^{X}$ denote the set of all $e \leq s$ such that the oracle Turing machine with index $e$ stops, when started with input $e$ and oracle $X$, after at most $s$ steps.

Next, let $F: \mathbb{N}^{3} \rightarrow\{0,1\}$ be defined by $F(e, m, s)=1$ if and only if the oracle Turing machine with index $e$ stops successfully after at most $s$ steps, when started with input $m$ and oracle $K_{s}^{\emptyset^{(n-2)}}$. Then $F$ is computable in $\emptyset^{(k-2)}$ and total. Let $A \in \Delta_{k}^{0}$. Then $A$ is computable in $\emptyset^{(k-1)}$, i.e., there exists $e \in \mathbb{N}$ such that the oracle Turing machine $M_{e}$ with index $e$ computes the characteristic function $\chi_{A}$ using the oracle $\emptyset^{(k-1)}$. Now let $m \in \mathbb{N}$. Then $M_{e}$ has a unique computation $C$ with input $m$ and oracle $\emptyset^{(k-1)}$. Let $t \in \mathbb{N}$ be at least the length of $C$ such that, for any oracle access $o$ in $C$, we have

$$
o \in \emptyset^{(k-1)}=K^{\emptyset^{(k-2)}} \Longleftrightarrow o \in K_{t}^{\emptyset^{(k-2)}} .
$$

Then, for any $s \geq t, C$ is the computation of the oracle Turing machine $M_{e}$ with oracle $K_{s}^{\emptyset^{(k-2)}}$ and input $m$. In other words, $F(e, m, s)=\chi_{A}(m)$ for any $s \geq t$. This proves (1) and (2).

Jockusch's first use of the limit lemma is in the base case:

LEMma 2.7. For any $n \geq 2$, we have $\left(\aleph_{0}, \Delta_{n-1}^{0}\right) \nrightarrow \rightarrow\left(\aleph_{0}, \Delta_{n}^{0}\right)_{2}^{2}$

Proof. Let $F$ be the function from Lemma 2.6 that is computable in $\emptyset^{(n-2)}$. We set $\{m, s\} \in E_{1}$ (with $m<s$ ) if and only if $m \in H(s)$ where $H(s)$ is defined by the procedure from Fig. $1 ; E_{2}=[\mathbb{N}]^{2} \backslash E_{1}$ completes the definition of the $(2,2)$ partition $\left(\mathbb{N}, E_{1}, E_{2}\right)$. Note that $E_{1}$ and $E_{2}$ are both computable in $\emptyset^{(n-2)}$ and therefore belong to $\Delta_{n-1}^{0}$.

We now show by contradiction that this partition does not have any infinite homogeneous set in $\Delta_{n}^{0}$. Let $A \in \Delta_{n}^{0}$ be infinite. Hence there is $e \in \mathbb{N}$ satisfying (1) 


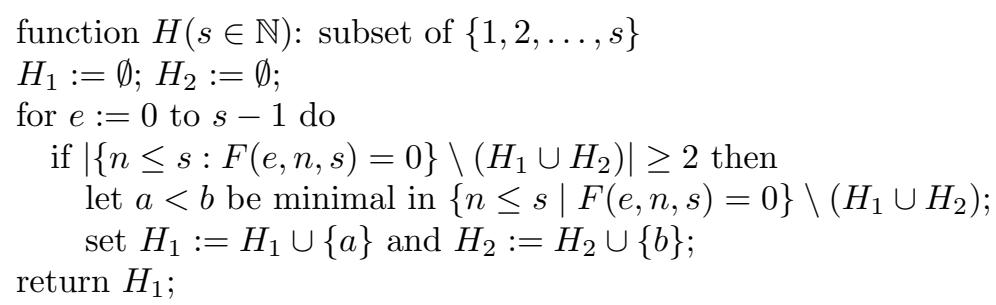

Figure 1. Computation of $H(s)$

and (2) in Lemma 2.6. Let $a_{1}<a_{2}<\cdots<a_{2 e+2}$ be the $2 e+2$ minimal elements of $A$. By Lemma 2.6, there is $t \in \mathbb{N}$ such that

$$
\forall a \leq a_{2 e+2} \forall s \geq t: a \in A \Longleftrightarrow F(e, a, s)=0 .
$$

Since $A$ is infinite, there exists $s \in A$ with $s \geq a_{2 e+2}$ and $s \geq t$. We analyse the set $H(s)$ : Note that in any run through the loop in Fig. 1, $\left|H_{1} \cup H_{2}\right|$ increases by at most 2 . Hence, whenever we execute line 3 , we get $\left|H_{1} \cup H_{2}\right| \leq 2 e$. With $e$ satisfying (1) and (2) in Lemma 2.6, the test in line 3 succeeds since $\{n \leq s$ : $F(e, n, s)=0\}=\left\{a_{1}, \ldots, a_{2 e+2}\right\}$ by $(2.1)$. For the same reason, the elements $a$ and $b$ chosen in line 4 both belong to $\left\{a_{1}, \ldots, a_{2 e+2}\right\} \subseteq A$. Since no element is ever deleted from $H_{1}$, we obtain $a \in H(s)$. On the other hand, no element from $H_{2}$ will ever be put into $H_{1}$, so $b \notin H(s)$. Hence, we found $a, b \in A$ with $\{a, s\} \in E_{1}$ and $\{b, s\} \in E_{2}$, i.e., the set $A$ is not homogeneous. Since all we assumed about the set $A$ was $A \in \Delta_{n}^{0}$ and $A$ infinite, we proved that no infinite $\Delta_{n}^{0}$-set is homogeneous in the (2,2)-partition $\left(\mathbb{N}, E_{1}, E_{2}\right)$.

By induction, Jockusch then increases the gap between $\Delta_{n-1}^{0}$ and $\Delta_{n}^{0}$ in the above lemma at the expense of partitioning $[\mathbb{N}]^{k}$ for $k>2$. This inductive argument makes use of the limit lemma, again.

Lemma 2.8. Let $P=(\mathbb{N}, A, B)$ be a $(k, 2)$-partition with $A, B \in \Delta_{n}^{0}$. Then there exists a $(k+1,2)$-partition $P^{\prime}=\left(\mathbb{N}, A^{\prime}, B^{\prime}\right)$ with $A^{\prime}, B^{\prime} \in \Delta_{n-1}^{0}$ such that any infinite set homogeneous in $P^{\prime}$ is also homogeneous in $P$.

Proof. Let $F$ be the function from Lemma 2.6 that is computable in $\emptyset^{(n-2)}$. Hence there is $e \in \mathbb{N}$ satisfying (1) and (2) in Lemma 2.6 (where we identify a finite set with its Gödel number). For $a_{1}<a_{2}<\cdots<a_{k}<s$, set $\left\{a_{1}, \ldots, a_{k}, s\right\} \in A^{\prime}$ if and only if $F\left(e,\left\{a_{1}, a_{2}, \ldots, a_{k}\right\}, s\right)=0$ and define $B^{\prime}=[\mathbb{N}]^{k+1} \backslash A^{\prime}$. Clearly, $A^{\prime}$ is computable in $\emptyset^{(n-2)}$ implying $A^{\prime}, B^{\prime} \in \Delta_{n-1}^{0}$.

Now let $H \subseteq \mathbb{N}$ be homogeneous in $P^{\prime}$. It suffices to consider the case $[H]^{k+1} \subseteq$ $A^{\prime}$ since the other one is analogous. To prove $[H]^{k} \subseteq A$, let $h_{1}<h_{2}<\cdots<h_{k}$ be elements of $H$. Then, for any $t>h_{k}$ from $H$, we get $F\left(e,\left\{h_{1}, \ldots, h_{k}\right\}, t\right)=0$. Since the sequence $\left(F\left(e,\left\{h_{1}, \ldots, h_{k}\right\}, s\right)\right)_{s \in \omega}$ is ultimately constant, this implies $F\left(e,\left\{h_{1}, \ldots, h_{k}\right\}, s\right)=0$ for almost all $s$, i.e., $\left\{h_{1}, \ldots, h_{k}\right\} \in A$.

THEOREM 2.9 (Jockusch [7]). Let $k, \ell, n \geq 2$. Then $\left(\aleph_{0}, \Delta_{n}^{0}\right) \nrightarrow \rightarrow\left(\aleph_{0}, \Sigma_{n+k-1}^{0}\right)_{\ell}^{k}$.

PROOF. We first prove $\left(\aleph_{0}, \Delta_{n}^{0}\right) \nrightarrow \rightarrow\left(\aleph_{0}, \Delta_{n+k-1}^{0}\right)_{2}^{k}$ :

By Lemma 2.7, we find a $(2,2)$-partition $P_{2}=\left(\mathbb{N}, E_{1}^{2}, E_{2}^{2}\right)$ with $E_{1}^{2}, E_{2}^{2} \in$ $\Delta_{n+k-2}^{0}$ without any homogeneous set in $\Delta_{n+k-1}^{0}$. Applying Lemma 2.8 several 
times, we find $(m, 2)$-partitions $P_{m}=\left(\mathbb{N}, E_{1}^{m}, E_{2}^{m}\right)$ with $E_{1}^{m}, E_{2}^{m} \in \Delta_{n+k-m}^{0}$ whose infinite homogeneous sets are also homogeneous in $P_{2}$ (and therefore do not belong to $\left.\Delta_{n+k-1}^{0}\right)$. With $m=k$, the claim follows.

The general result now follows since:

- Any infinite set $H$ from $\Sigma_{n+k-1}^{0}$ contains an infinite set from $\Delta_{n+k-1}^{0}$ that is homogeneous whenever $H$ is.

- If $\ell>2$, extend the $(n, 2)$-partition by sufficiently many empty classes.

Let $P=\left(\mathbb{N}, E_{1}, E_{2}\right)$ be some infinite computable $(2,2)$-partition. Let $R=$ $(\mathbb{N}, \preceq)$ be its Ramsey tree (with respect to the natural order $\leq$ on $\mathbb{N}$ ) and suppose $C \subseteq \mathbb{N}$ is an infinite chain in $R$ from $\Sigma_{2}^{0}$. Then the sets $E_{i}^{C}$ of natural numbers $c \in C$ with

$$
\exists d: d \in C \wedge c<d \wedge\{c, d\} \in E_{i}
$$

belong to $\Sigma_{2}^{0}$ and are homogeneous in $P^{C}$ and therefore in $P$. Furthermore, one of $E_{1}^{C}$ and $E_{2}^{C}$ is infinite. But this contradicts $\left(\aleph_{0}, \Delta_{1}^{0}\right) \not \rightarrow\left(\aleph_{0}, \Sigma_{2}^{0}\right)_{2}^{2}$. Hence the Ramsey tree of $P$ does not necessarily contain an infinite chain from $\Sigma_{2}^{0}$, i.e., we proved

COROLlARY 2.10. There exists a computable finitely branching successor tree without any infinite chain from $\Sigma_{2}^{0}$.

2.3. Rubin's theorem. Rubin [15] considered Ramsey's theorem in the context of automatic structures $[\mathbf{9}, \mathbf{3}]$. While a computable structure is given by a tuple of Turing machines, an automatic structure is given by a tuple of finite automata. We therefore first sketch some basic notions from the theory of finite automata.

2.3.1. Languages of finite words. Let $\Gamma$ be some finite alphabet. The set of all (finite) words over $\Gamma$ is denoted $\Gamma^{*}$, the empty word is $\varepsilon$, and $\Gamma^{+}$is the set of non-empty words. By $\leq_{\text {pref }}$, we denote the prefix order on $\Gamma^{*}$.

A finite automaton $M$ is a tuple $M=(Q, \Gamma, \delta, I, F)$ where $Q$ is a finite set of states, $I \subseteq Q$ is the set of initial states, $F \subseteq Q$ is the set of final states, and $\delta \subseteq Q \times \Gamma \times Q$ is the transition relation. A run of $M$ on a word $x=a_{1} a_{2} \cdots a_{n}$ with $n \geq 0$ and $a_{i} \in \Gamma$ is a word $q_{0} q_{1} \ldots q_{n} \in Q^{+}$with $q_{i} \in Q$ such that $\left(q_{i}, a_{i+1}, q_{i+1}\right) \in \delta$ for all $0 \leq i<n$. The run is successful if $q_{0} \in I$ and $q_{n} \in F$. The language $L(M) \subseteq \bar{\Gamma}^{*}$ defined by $M$ is the set of all words that admit a successful run. A language $L \subseteq \Gamma^{*}$ is regular if there exists a finite automaton $M$ with $L(M)=L$. By REG, we denote the class of all regular languages.

For words $x_{i}=a_{i}^{0} a_{i}^{1} a_{i}^{2} \ldots a_{i}^{k_{i}} \in \Gamma^{*}$, the convolution $\left(x_{1}, x_{2}, \ldots, x_{n}\right)^{\otimes} \in((\Gamma \uplus$ $\left.\{\#\})^{n}\right)^{*}$ is defined by

$$
\left(x_{1}, \ldots, x_{n}\right)^{\otimes}=\left(b_{1}^{0}, \ldots, b_{n}^{0}\right)\left(b_{1}^{1}, \ldots, b_{n}^{1}\right)\left(b_{1}^{2}, \ldots, b_{n}^{2}\right) \cdots\left(b_{1}^{k}, \ldots, b_{n}^{k}\right)
$$

where $k=\max \left\{k_{1}, \ldots, k_{n}\right\}$ and

$$
b_{i}^{\ell}= \begin{cases}a_{i}^{\ell} & \text { if } \ell \leq k_{i} \\ \# & \text { otherwise }\end{cases}
$$

In other words, the convolution is obtained by first adding some occurrences of the new letter \# to each word to make them the same length and then "glueing" the prolonged words together. An $n$-ary relation $R \subseteq\left(\Gamma^{*}\right)^{n}$ is called automatic if the language $\left\{\left(x_{1}, \ldots, x_{n}\right)^{\otimes} \mid\left(x_{1}, \ldots, x_{n}\right) \in R\right\}$ is regular. In order to also capture 
partition classes of a $(k, \ell)$-partition, we will call a set $E \subseteq\left[\Gamma^{*}\right]^{k}$ automatic if the relation

$$
\left\{\left(u_{1}, \ldots, u_{n}\right) \mid\left\{u_{1}, \ldots, u_{n}\right\} \in E\right\}
$$

is automatic, i.e., if the language

$$
\left\{\left(u_{1}, \ldots, u_{n}\right)^{\otimes} \mid\left\{u_{1}, \ldots, u_{n}\right\} \in E\right\}
$$

is regular. We write $A$ for the class of automatic subsets of $\left[\Gamma^{*}\right]^{k}$ for any $k \in \mathbb{N}$ and alphabet $\Gamma$.

2.3.2. Ramsey's theorem for automatic partitions. Note that any partition class from $\mathrm{A}$ is decidable. Hence, any $(k, \ell)$-partition with automatic partition classes is computable and therefore has an infinite homogeneous set in the arithmetical hierarchy. Rubin showed that in this case, much simpler infinite homogeneous sets can be found. Namely, he proved

Proposition 2.11 (Rubin [15]). Let $\left(V, E_{1}, \ldots, E_{\ell}\right)$ be some $(k, \ell)$-partition with $E_{i} \in \mathrm{A}$ for all $1 \leq i \leq \ell$ that has some infinite $E_{1}$-homogeneous set. Then there is some infinite regular $E_{1}$-homogeneous set.

Since every infinite partition has, by Ramsey's theorem, some homogeneous set, we get immediately:

Theorem 2.12 (Rubin [15, Prop. 3.21]). For all $k, \ell \geq 1$, we have

$$
\left(\aleph_{0}, A\right) \rightarrow\left(\aleph_{0}, A\right)_{\ell}^{k}
$$

Since $L \subseteq \Gamma^{*}$ is automatic if and only if it is regular, we can alternatively express this as

$$
\left(\aleph_{0}, \mathrm{~A}\right) \rightarrow\left(\aleph_{0}, \mathrm{REG}\right)_{\ell}^{k}
$$

The proof of Prop. 2.11 does not use the Ramsey tree of the partition for two reasons. First, the proof is simpler since it constructs the regular homogeneous set directly. Secondly, it is not clear whether the Ramsey tree of an automatic partition is automatic, i.e., can be described by finite automata. Hence, we do not know how to perform the induction in the automatic setting.

The direct construction of the regular homogeneous set in Prop. 2.11 uses the concept of a comb: a comb is a set of words $C=\left\{s_{0} s_{1} s_{2} \ldots s_{i-1} t_{i} \mid 0 \leq i<\alpha\right\}$ for some $\alpha \leq \omega$ where $s_{i}, t_{i} \in \Gamma^{+}$and $\left|t_{i}\right|=\left|s_{i}\right|$ for all $0 \leq i<\alpha$ (note that, given $C$, these conditions determine $s_{i}$ and $t_{i}$ completely). A comb $C$ is visualized in Fig. 2: it consists of the paths from the empty node to the filled nodes since these paths are labeled $t_{0}, s_{0} t_{1}, s_{0} s_{1} t_{2}, \ldots, s_{0} s_{1} s_{2} s_{3} t_{4}$.

Given a $(k, \ell)$-partition $P=\left(V, E_{1}, \ldots, E_{\ell}\right)$ with $E_{i}$ automatic, our first lemma ensures that any infinite homogeneous set contains some infinite comb:

Lemma 2.13. Let $X \subseteq \Gamma^{*}$ be infinite. Then there exists an infinite comb $C \subseteq X$

Proof. Let $t_{0} \in X \backslash\{\varepsilon\}$ be arbitrary. Now suppose we defined $s_{0}, \ldots, s_{j-1}$ and $t_{0}, \ldots, t_{j}$ such that

(1) $s_{0} s_{1} \ldots s_{i-1} t_{i} \in X$ for all $0 \leq i \leq j$

(2) $\left|s_{i}\right|=\left|t_{i}\right|$ for all $0 \leq i<j$

(3) $X \cap s_{0} s_{1} \ldots s_{j-1} \Gamma^{+}$is infinite. 


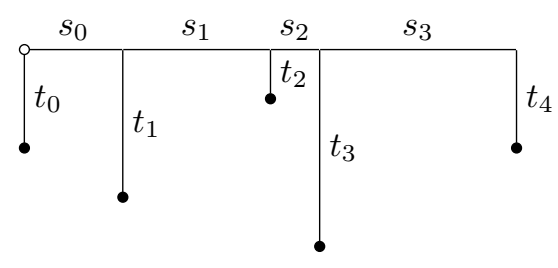

\begin{tabular}{|c|c|c|c|c|}
\hline$s_{0}$ & $s_{1}$ & $s_{2}$ & $s_{3}$ & $s_{4}$ \\
\hline$t_{0}$ & $t_{1}$ & $t_{2}$ & $t_{3}$ & $t_{4}$ \\
\hline 00001 & 00000001 & 01 & 00000000001 & 00001 \\
\hline
\end{tabular}

Figure 2. A comb and its coding

Since there are only finitely many words of length $\left|t_{j}\right|$, (3) implies the existence of a word $s_{j} \in \Gamma^{+}$with $\left|s_{j}\right|=\left|t_{j}\right|$ such that $X \cap s_{0} s_{1} \ldots s_{j} \Gamma^{+}$is infinite ensuring (2) and (3) for $j+1$. Choose $t_{j+1} \in \Gamma^{+}$with $s_{0} s_{1} \ldots s_{j} t_{j+1} \in X$ arbitrary. Since this ensures (1), we can proceed by induction. Then the set of words $s_{0} s_{1} \ldots s_{i-1} t_{i}$ is an infinite comb $C \subseteq X$.

Words from $\left(\Sigma^{2} \times\{0,1\}\right)^{*}$ encode finite combs as follows:

Definition 2.14. Let $n_{0}, n_{1}, \ldots, n_{\alpha} \in \mathbb{N}$ and $w=0^{n_{0}} 10^{n_{1}} 1 \ldots 0^{n_{\alpha-1}} 10^{n_{\alpha}}$. Furthermore, let $s_{i}, t_{i} \in \Sigma^{*}$ with $\left|s_{i}\right|=\left|t_{i}\right|=n_{i}+1$ for $0 \leq i<\alpha$ and $\left|s_{\alpha}\right|=$ $\left|t_{\alpha}\right|=n_{\alpha}$. The comb encoded by $\left(s_{0} s_{1} \ldots s_{\alpha}, t_{0} t_{1} \ldots t_{\alpha}, w\right)^{\otimes}$ is the set of words $\left\{s_{0} s_{1} \ldots s_{i-1} t_{i} \mid 0 \leq i \leq \alpha\right\}$.

Note that any word from $\left(\Sigma^{2} \times\{0,1\}\right)^{*}$ determines $\alpha, n_{i}, s_{i}$, and $t_{i}$ and therefore encodes a unique comb. Conversely, every finite comb can be encoded by some worf from $\left(\Sigma^{2} \times\{0,1\}\right)^{*}$ (which is not unique since $s_{\alpha}$ is arbitrary).

Lemma 2.15. Let $E \subseteq\left[\Sigma^{*}\right]^{k}$ be automatic. Then the set of encodings of finite E-homogeneous combs is regular.

Proof. Since the class of regular languages is closed under complementation, it suffices to construct a finite automaton that accepts a word from $\left(\Sigma^{2} \times\{0,1\}\right)^{*}$ if and only if it does not encode an $E$-homogeneous comb.

So let $M=\left(Q,(\Sigma \cup\{\#\})^{k}, \delta, I, F\right)$ be a finite automaton that accepts the set $E^{\otimes}=\left\{\left(u_{1}, \ldots, u_{k}\right)^{\otimes} \mid\left\{u_{1}, \ldots, u_{k}\right\} \in E\right\}$. We can assume that $M$ is deterministic and complete, i.e., $|I|=1$ and for any $p \in Q$ and $a \in(\Sigma \cup\{\#\})^{k}$, there exists a unique state $q \in Q$ with $(p, a, q) \in \delta$. We build a finite automaton $M^{\prime}$ as follows:

- states of $M^{\prime}$ are pairs $(q, f)$ with $q \in Q, f:\{1, \ldots, k\} \rightarrow\{1,2,3\}$, and $\left|f^{-1}(2)\right| \leq 1$,

- a state $(q, f)$ is initial if and only if $q \in I$ and $f(i) \leq 2$ for all $1 \leq i \leq k$,

- a state $(q, f)$ is accepting (i.e., belongs to $\left.F^{\prime}\right)$ if and only if $q \notin F$ and $f(i) \geq 2$ for all $1 \leq i \leq k$, and

- a triple $\left((q, f),(a, b, x),\left(q^{\prime}, f^{\prime}\right)\right) \in Q^{\prime} \times\left(\Sigma^{2} \times\{0,1\}\right) \times Q^{\prime}$ is a transition from $\delta^{\prime}$ if and only if

$-f(i) \leq f^{\prime}(i) \leq f(i)+x$ for all $1 \leq i \leq k$ and 
$-\left(q,\left(c_{i}\right)_{1 \leq i \leq k}, q^{\prime}\right) \in \delta$ where

$$
c_{i}= \begin{cases}a & \text { if } f(i)=1 \\ b & \text { if } f(i)=2 \\ \# & \text { otherwise. }\end{cases}
$$

Let $(s, t, w)^{\otimes} \in\left(\Sigma^{*} \times\{0,1\}\right)^{*}$. We use $\alpha, n_{i}, s_{i}$, and $t_{i}$ as in Definition 2.14. Then the automaton $M^{\prime}$ has a run from some initial state to $(q, f)$ labeled $(s, t, w)^{\otimes}$ if and only if there are pairwise distinct numbers $0 \leq j_{i}<\alpha$ for $f(i)>1$ and a run of $M$ from $\iota \in I$ to $q$ labeled $\left(u_{1}, \ldots, u_{k}\right)^{\otimes}$ where

(1) $u_{i}= \begin{cases}s & \text { if } f(i)=1 \\ s_{0} s_{1} \ldots s_{j_{i}} t_{j_{i}+1} & \text { if } f(i)>1,\end{cases}$

(2) $j_{i}=\alpha-1$ implies $f(i)=2$ or $n_{\alpha}=0$, and

(3) $f(i)=2$ implies $j_{i}=\alpha-1$.

Now suppose $(s, t, w)^{\otimes} \in\left(\Sigma^{2} \times\{0,1\}\right)^{*}$. Then $(s, t, w)^{\otimes}$ is accepted by $M^{\prime}$ if and only if there is some $q \in Q \backslash F$ such that $M^{\prime}$ has a run from some initial state to $(q, f)$ labeled $(s, t, w)^{\otimes}$ where $f(i)=3$ for all $i$. This is, by the above observation, equivalent to the existence of pairwise distinct $0 \leq j_{i}<\alpha$ for $1 \leq i \leq k$ (where $j_{i}=\alpha-1$ is only allowed if $n_{\alpha}=0$ ) and a run of $M$ from $\iota \in I$ to $q$ labeled $\left(u_{1}, \ldots, u_{k}\right)^{\otimes}$ where $u_{i}=s_{0} s_{1} \ldots s_{j_{i}} t_{j_{i}+1}$ for $1 \leq i \leq k$. Since $M$ is deterministic and complete, such a run exists if and only if $\left\{u_{1}, \ldots, u_{k}\right\} \notin E$. Hence, indeed, the finite automaton $M^{\prime}$ accepts a word $(s, t, w)^{\otimes}$ from $\left(\Sigma^{2} \times\{0,1\}\right)^{*}$ if and only if it encodes a comb that is not $E$-homogeneous.

Proof of Prop. 2.11. Let $\left(V, E_{1}, \ldots, E_{\ell}\right)$ be an infinite $(k, \ell)$-partition with $E_{i}$ automatic for all $1 \leq i \leq \ell$ and let $X \subseteq V$ by $E_{1}$-homogeneous. By Lemma 2.13, there exists an infinite comb $C \subseteq X$ (that is also $E_{1}$-homogeneous). Let $C=$ $\left\{s_{0} s_{1} s_{2} \ldots s_{i-1} t_{i} \mid 0 \leq i<\omega\right\}$ with $s_{i}, t_{i} \in \Gamma^{+}$and $\left|t_{i}\right|=\left|s_{i}\right|$ for all $0 \leq i<\omega$. For $0<\alpha<\omega$, let $W_{\alpha}$ encode the finite comb $\left\{s_{0} s_{1} s_{2} \ldots s_{i-1} t_{i} \mid 0 \leq i<\alpha\right\}$. We can assume $W_{1}<$ pref $W_{2}<$ pref $W_{3} \ldots$

Let $L$ consist of all words from $\left(\Sigma^{2} \times\{0,1\}\right)^{*}\left(\Sigma^{2} \times\{1\}\right)$ that encode some $E_{1}$-homogeneous comb. Since $L$ is regular by Lemma 2.15 , it can be accepted by a deterministic and complete finite automaton. Hence there are $m<n$ and a word $W$ with $W_{n}=W_{m} W$ such that $W_{m} W^{*} \subseteq L$ (we write $V$ for $W_{m}$ such that $V W^{*} \subseteq L$ ).

There are words $s_{V}, t_{V}, s_{W}, t_{W} \in \Sigma^{+}$and $w_{V}, w_{W} \in\{0,1\}^{+}$such that $V=$ $\left(s_{V}, t_{V}, w_{V}\right)^{\otimes}$ and $W=\left(s_{W}, t_{W}, w_{W}\right)^{\otimes}$. Let $n$ be such that $0^{n} 1$ is a prefix of $w_{W}$ and let $t$ be the prefix of $t_{W}$ of length $n+1$.

Since $V \in L$, the word $w_{V}$ ends with 1 . For $1 \leq i \leq k$, let $u_{i}=s_{V} s_{W}^{n_{i}} t$ for pairwise distinct numbers $n_{i}$ and let $\alpha<\omega$ be some upper bound for the numbers $n_{i}$. Then all the words $u_{i}$ belong to the comb encoded by $V W^{\alpha} \in L$. Since this comb is $E_{1}$-homogeneous, we get $\left[s_{V} s_{W}^{+} t\right]^{k} \subseteq E_{1}$. Hence, indeed, there is some infinite regular homogeneous set.

The concept of a comb was first used by Khoussainov et al. in [10] where they show that every automatic order tree with at least one infinite branch has a regular infinite branch. Büchi exploited the relation between automata and logic and showed, in this context, that the first-order theory of every automatic structure is decidable [9]. Rubin [15] proved, using combs again, that this holds even if firstorder logic is extended by Ramsey quantifiers. This result was then extended by 
Kuske \& Lohrey [12] to a weak form of second-order quantification, namely to quantifications of the form

$$
\exists R \text { infinite }: \varphi
$$

where the relational variable $R$ occurs only negatively in $\varphi$. This decidability implies that certain graph problems that are $\Sigma_{1}^{1}$-complete for computable graphs are decidable for automatic graphs $[\mathbf{1 2}]$.

The original proofs of all these results used infinite combs and therefore Büchiautomata that accept infinite words. Differently, the above proof works in the realm of finite automata and finite words.

\section{The uncountable case}

We now deal with the existence of uncountable homogeneous sets. The first observation in this direction is due to Sierpiński who showed that the direct analogue for Ramsey's theorem $\aleph_{0} \rightarrow\left(\aleph_{0}\right)_{\ell}^{k}$ does not hold:

THEOREM 3.1 (Sierpiński $[\mathbf{1 7}])$. If $k, \ell \geq 2$, then $2^{\aleph_{0}} \nrightarrow \rightarrow\left(\aleph_{1}\right)_{\ell}^{k}$ and therefore in particular $2^{\aleph_{0}} \not \rightarrow\left(2^{\aleph_{0}}\right)_{\ell}^{k}$.

PROOF. We first sketch the proof of $2^{\aleph_{0}} \nrightarrow \rightarrow\left(\aleph_{1}\right)_{2}^{2}$ : Let $\sqsubseteq$ be some well-order on $\mathbb{R}$ and let $E_{1}=\left\{\{x, y\} \in[\mathbb{R}]^{2} \mid x \leq y \Longleftrightarrow x \sqsubseteq y\right\}$ and $E_{2}=[\mathbb{R}]^{2} \backslash E_{1}$. If $H \subseteq \mathbb{R}$ is $E_{1}$-homogeneous, then $(H, \sqsubseteq)=(H, \leq) \hookrightarrow(\mathbb{R}, \leq)$, i.e., the wellorder $(H, \sqsubseteq)$ embeds into the linear order $(\mathbb{R}, \leq)$. Hence $H$ is countable. If $H$ is $E_{2}$-homogeneous, then $(H, \sqsubseteq)=(H, \geq) \hookrightarrow(\mathbb{R}, \geq) \cong(\mathbb{R}, \leq)$, so again $H$ is countable. Hence the $(2,2)$-partition $\left(\mathbb{R}, E_{1}, E_{2}\right)$ does not contain any uncountable homogeneous set.

To also show $2^{\aleph_{0}} \not \rightarrow\left(\aleph_{1}\right)_{\ell}^{k}$ for $k>2$, define $A \in E_{i}^{\prime}$ if and only if $\left\{x_{1}, x_{2}\right\} \in E_{i}$ where $x_{1}<x_{2}$ are the two minimal elements of $A$. Then $\left(\mathbb{R}, E_{1}^{\prime}, E_{2}^{\prime}, \emptyset, \ldots, \emptyset\right)$ is a $(k, \ell)$-partition of size $2^{\aleph_{0}}$ without uncountable homogeneous sets.

3.1. Erdős \& Rado's theorem. This theorem is concerned with homogeneous sets of size $\aleph_{1}$. We will assume the generalized continuum hypothesis $2^{\aleph_{k}}=\aleph_{k+1}$ for all $k \in \omega$.

Theorem 3.2 (Erdös \& Rado [6]). For all $k, \ell \geq 1$, we have

$$
\aleph_{k} \rightarrow\left(\aleph_{1}\right)_{\ell}^{k}
$$

Proof. The proof is, as the proof of Ramsey's theorem, by induction on $k$ where the base case $k=1$ is trivial.

So let $P=\left(V, E_{1}, \ldots, E_{\ell}\right)$ be some $(k+1, \ell)$-partition of size $\aleph_{k+1}$ and consider the Ramsey tree of $P$ with respect to some well-order on $V$. Recall that every node at level $\alpha<\omega_{k}$ has at most $2^{\aleph_{k-1}}=\aleph_{k}$ brothers. Hence, by induction, there are at most $\aleph_{k}$ nodes at level $\alpha$. In total, there are only $\aleph_{k}$ nodes on levels $<\omega_{k}$. Since the size of the Ramsey tree equals that of the $(k+1, \ell)$-partition, there is at least one node at level $\omega_{k}$. So the Ramsey tree contains a chain $Y$ of size at least $\aleph_{k}$. Let $P^{Y}$ be the $(k, \ell)$-partition induced by $Y$. Since, by induction, we have $\aleph_{k} \rightarrow\left(\aleph_{1}\right)_{\ell}^{k}$, the $(k, \ell)$-partition $P^{Y}$ contains some homogeneous set of size $\aleph_{1}$. But this set is also homogeneous in $P$ by Prop. 1.4.

3.2. Ramsey's theorem for $\omega$-automatic partitions. We next want to find an effective analogue of the theorem of Erdös and Rado. For this, we use the framework of $\omega$-automatic structures as defined formally in $[\mathbf{2}, \mathbf{3}]$. 
3.2.1. Languages of infinite words. An $\omega$-word over the alphabet $\Gamma$ is an infinite $\omega$-sequence $x=a_{0} a_{1} a_{2} \cdots$ with $a_{i} \in \Gamma$. The set of all $\omega$-words over $\Gamma$ is denoted by $\Gamma^{\omega}$. For a set $V \subseteq \Gamma^{+}$of finite words let $V^{\omega} \subseteq \Gamma^{\omega}$ be the set of all $\omega$-words of the form $v_{0} v_{1} v_{2} \cdots$ with $v_{i} \in V$.

Two $\omega$-words $x$ and $y$ are eventually equal (denoted $x \sim_{e} y$ ) if, from some position on, the two words coincide. For $\Sigma=\{0,1\}$, the $\operatorname{support} \operatorname{supp}(x) \subseteq \mathbb{N}$ is the set of positions of the letter 1 in the word $x \in \Sigma^{\omega}$.

A run of a finite automaton $M=(Q, \Gamma, \delta, I, F)$ on an $\omega$-word $x=a_{0} a_{1} a_{2} \cdots$ is an $\omega$-word $r=p_{0} p_{1} p_{2} \cdots$ over the set of states $Q$ such that $\left(p_{i}, a_{i}, p_{i+1}\right) \in \delta$ for all $i \geq 0$. The run $r$ is successful if $p_{0} \in I$ and there exists a final state from $F$ that occurs infinitely often in $r$. The $\omega$-language $L^{\omega}(M) \subseteq \Gamma^{\omega}$ defined by $M$ is the set of all $\omega$-words that admit a successful run. Note that a finite automaton $M$ accepts a language of finite words $L(M)$ and an $\omega$-language $L^{\omega}(M)$. Whenever we are more interested in the latter, we will speak of $M$ as a Büchi-automaton. An $\omega$-language $L \subseteq \Gamma^{\omega}$ is regular if there exists a Büchi-automaton $M$ with $L^{\omega}(M)=L$.

Alternatively, regular $\omega$-languages can be represented algebraically. To this end, one defines $\omega$-semigroups to be two-sorted algebras $S=\left(S_{+}, S_{\omega} ; \cdot, *, \pi\right)$ where $\cdot: S_{+} \times S_{+} \rightarrow S_{+}$and $*: S_{+} \times S_{\omega} \rightarrow S_{\omega}$ are binary operations and $\pi:\left(S_{+}\right)^{\omega} \rightarrow S_{\omega}$ is an $\omega$-ary operation such that the following hold:

- $\left(S_{+}, \cdot\right)$ is a semigroup,

- $s *(t * u)=(s \cdot t) * u$,

- $s_{0} \cdot \pi\left(\left(s_{i}\right)_{i \geq 1}\right)=\pi\left(\left(s_{i}\right)_{i \geq 0}\right)$,

- $\pi\left(\left(s_{i}^{1} \cdot s_{i}^{2} \cdots s_{i}^{k_{i}}\right)_{i \geq 0}\right)=\pi\left(\left(t_{j}\right)_{j \geq 0}\right)$ whenever

$$
\left(t_{j}\right)_{j \geq 0}=\left(s_{0}^{1}, s_{0}^{2}, \ldots, s_{0}^{k_{0}}, s_{1}^{1}, \ldots, s_{1}^{k_{1}}, \ldots\right) .
$$

The $\omega$-semigroup $S$ is finite if both, $S_{+}$and $S_{\omega}$ are finite. The free $\omega$-semigroup generated by $\Gamma$ is

$$
\Gamma^{\infty}=\left(\Gamma^{+}, \Gamma^{\omega} ; \cdot, *, \pi\right)
$$

where $u \cdot v$ and $u * x$ are the natural operations of prefixing a word by the finite word $u$, and $\pi\left(\left(u_{i}\right)_{i>0}\right)$ is the $\omega$-word $u_{0} u_{1} u_{2} \ldots$ A homomorphism $h: \Gamma^{\infty} \rightarrow S$ of $\omega$-semigroups maps finite words to elements of $S_{+}$and $\omega$-words to elements of $S_{\omega}$ and commutes with the operations $\cdot, *$, and $\pi$. The algebraic characterisation of regular $\omega$-languages then reads as follows.

Proposition 3.3. An $\omega$-language $L \subseteq \Gamma^{\omega}$ is regular if and only if there exists a finite $\omega$-semigroup $S$ and a homomorphism $\eta: \Gamma^{\infty} \rightarrow S$ such that $L=\eta^{-1}(\eta(L))$.

Hence, every Büchi-automaton $M$ is "equivalent" to a homomorphism into some finite $\omega$-semigroup together with a distinguished set $\eta\left(L^{\omega}(M)\right)$ (and vice versa). Even more, this translation is effective - see [13] for this and more results on regular $\omega$-languages. Note that the equation $L=\eta^{-1}(\eta(L))$ can also be read as

$$
\eta(x)=\eta(y) \Longrightarrow(x \in L \Longleftrightarrow y \in L)
$$

for all $\omega$-words $x$ and $y$.

For $\omega$-words $x_{i}=a_{i}^{0} a_{i}^{1} a_{i}^{2} \cdots \in \Gamma^{\omega}$, the convolution $x_{1} \otimes x_{2} \otimes \cdots \otimes x_{n} \in\left(\Gamma^{n}\right)^{\omega}$ is defined by

$$
\left(x_{1}, \ldots, x_{n}\right)^{\otimes}=\left(a_{1}^{0}, \ldots, x_{n}^{0}\right)\left(a_{1}^{1}, \ldots, a_{n}^{1}\right)\left(a_{1}^{2}, \ldots, a_{n}^{2}\right) \cdots .
$$


An $n$-ary relation $R \subseteq\left(\Gamma^{\omega}\right)^{n}$ is called $\omega$-automatic if the $\omega$-language $\left\{\left(x_{1}, \ldots, x_{n}\right)^{\otimes} \mid\right.$ $\left.\left(x_{1}, \ldots, x_{n}\right) \in R\right\}$ is regular, a partition class $E \subseteq\left[\Gamma^{\omega}\right]^{k}$ is $\omega$-automatic if the relation $\left\{\left(x_{1}, \ldots, x_{k}\right) \mid\left\{x_{1}, \ldots, x_{k}\right\} \in E\right\}$ is.

To describe the complexity of $\omega$-languages, we will use language-theoretic terms. Let $\omega$ LANG denote the class of all $\omega$-languages (i.e., sets of $\omega$-words over some finite set of symbols). By $\omega \mathrm{REG}$, we denote the regular $\omega$-languages. A language is context-free if it can be accepted by a pushdown-automaton. An $\omega$-language is eventually regular context-free if it is a finite union of $\omega$-languages $U V$ with $U$ context-free and $V \in \omega \mathrm{REG}$. Let co-werCF denote the set of complements of eventually regular context-free $\omega$-languages.

A final, rather peculiar class of $\omega$-languages is $\boldsymbol{\Lambda}$ : it is the class of $\omega$-languages $L$ such that $(\mathbb{R}, \leq)$ embeds into $\left(L, \leq_{\text {lex }}\right)$ (the name derives from the notation $\lambda$ for the order type of $(\mathbb{R}, \leq))$.

An $\omega$-automatic presentation of a $(k, \ell)$-partition $\left(V, E_{1}, \ldots, E_{\ell}\right)$ is a pair $(L, h)$ consisting of an $\omega$-language $L$ and a surjection $h: L \rightarrow V$ such that

$$
\begin{aligned}
& E_{i}^{\otimes}=\left\{\left(x_{1}, x_{2}, \ldots, x_{k}\right) \in L^{k} \mid\left\{h\left(x_{1}\right), h\left(x_{2}\right), \ldots, h\left(x_{k}\right)\right\} \in E_{i}\right\} \text { for } 1 \leq i \leq k \text { and } \\
& R_{\approx}=\left\{\left(x_{1}, x_{2}\right) \in L^{2} \mid h\left(x_{1}\right)=h\left(x_{2}\right)\right\}
\end{aligned}
$$

are $\omega$-automatic. An $\omega$-automatic presentation is injective if $h$ is a bijection. A $(k, \ell)$-partition is (injectively) $\omega$-automatic if it has an (injective) $\omega$-automatic presentation. From [1] , it follows that an uncountable $\omega$-automatic $(k, \ell)$-partition has $2^{\aleph_{0}}$ elements.

Extending notions we used before, we write

$$
(\kappa, \omega \mathrm{A}) \rightarrow(\lambda, \mathcal{C})_{\ell}^{k}
$$

if the following partition property holds: for every $\omega$-automatic presentation $(L, h)$ of a $(k, \ell)$-partition $P$ of size $\kappa$, there exists $H \subseteq L$ in $\mathcal{C}$ such that $h(H)$ is homogeneous in $P$ and of size $\lambda$;

$$
(\kappa, \omega \mathrm{iA}) \rightarrow(\lambda, \mathcal{C})_{\ell}^{k}
$$

is to be understood similarly where we only consider injective $\omega$-automatic presentations.

REMARK 3.4. Let $P=\left(V, E_{1}, \ldots, E_{\ell}\right)$ be some $(k, \ell)$-partition with $\omega$-automatic presentation $(L, h)$. Then the partition property above requires that there is a "large" homogeneous set $X \subseteq V$ and an $\omega$-language $H \in \mathcal{C}$ such that $h(H)=X$, in particular, every element of $X$ has at least one representative in $H$. Alternatively, one could require that $h^{-1}(X) \subseteq L$ is an $\omega$-language from $\mathcal{C}$. We will only encounter classes $\mathcal{C}$ of $\omega$-languages such that the following closure property holds: if $H \in \mathcal{C}$ and $R$ is an $\omega$-automatic relation, then also $R(H)=\{y \mid \exists x \in H:(x, y) \in R\} \in \mathcal{C}$. Since $h^{-1} h(H)=R_{\approx}(H)$, all our results also hold for this alternative requirement $h^{-1}(X) \in \mathcal{C}$.

\subsubsection{Ramsey's theorem for $\omega$-automatic partitions. We want to prove}

$$
\left(2^{\aleph_{0}}, \omega \mathrm{A}\right) \rightarrow\left(2^{\aleph_{0}}, \mathrm{co}-\omega \mathrm{erCF} \cap \boldsymbol{\Lambda}\right)_{\ell}^{2}
$$

for all $\ell \geq 2$. Hence we will be concerned with edge-labeled graphs. The proof will not be done via the Ramsey tree. Instead, we will identify a common substructure of all $\omega$-automatic edge-labeled graphs (see Example 3.5) and show that this substructure gives rise to homogeneous sets as required. 
ExAmple 3.5. Let $V=\{0,1\}^{\omega}$ consist of all $\omega$-words. Two distinct such words $x$ and $y$ are connected by an edge (i.e., $\{x, y\}$ belongs to $E_{1}$ ) if and only if $\operatorname{supp}(x) \backslash \operatorname{supp}(y)$ and $\operatorname{supp}(y) \backslash \operatorname{supp}(x)$ are both infinite; $E_{2}=[V]^{2} \backslash E_{1}$ denotes the set of non-edges. We construct an instructive $E_{1}$-homogeneous set of size $2^{\aleph_{0}}$. For this, let $u_{0}, u_{1}, u_{2}, \ldots$ be the list of words from $\{0,1\}^{*}$ in length-lexicographic order (i.e., $u$ comes before $v$ if $|u|<|v|$ or $|u|=|v|, u=x 0 y$ and $v=x 1 z$ ). Intuitively, we list the nodes of the complete binary tree $\left(\{0,1\}^{*}, \leq_{\text {pref }}\right)$ level-wise where each level is listed from left to right. Let the $\omega$-language $N$ consist of all $\omega$-words $x \in\{0,1\}^{\omega}$ such that

$$
\left\{u_{i} \mid i \in \operatorname{supp}(x)\right\}
$$

is an infinite branch in the complete binary tree $\left(\{0,1\}^{*}, \leq_{\text {pref }}\right)$. Then, clearly, $N \subseteq V$ has size $2^{\aleph_{0}}$ since its elements correspond to infinite branches. An $\omega$-word $x \in V$ does not belong to $N$ if and only if

- $0 \notin \operatorname{supp}(x)$ or

- $\exists i \in \operatorname{supp}(x):|\{2 i+1,2 i+2\} \cap \operatorname{supp}(x)| \neq 1$ or

- $\exists i \in \operatorname{supp}(x) \backslash\{0\}:\left\lfloor\frac{i-1}{2}\right\rfloor \notin \operatorname{supp}(x)$.

Since this condition relates positions $i$ and $2 i$, this is a typical context-free condition, hence $N$ is the complement of an eventually regular context-free language. Finally, any two branches have a finite intersection, $\operatorname{so} \operatorname{supp}(x) \cap \operatorname{supp}(y)$ is finite for any $x, y \in N$ distinct. $\operatorname{Since} \operatorname{supp}(x)$ and $\operatorname{supp}(y)$ are infinite, this implies $[N]^{2} \subseteq E_{1}$. Hence we found an $E_{1}$-homogeneous set of size $2^{\aleph_{0}}$ whose complement is eventually regular context-free. Let $x, y \in N$ be distinct with $X=\left\{u_{i} \mid i \in \operatorname{supp}(x)\right\}$ and $Y=\left\{u_{i} \mid i \in \operatorname{supp}(y)\right\}$. Then $x<_{\text {lex }} y$ if and only if the branch $X$ is right of the branch $Y$. Since $(\mathbb{R}, \leq)$ can be embedded into the set of branches (ordered naturally from right to left), there is also an embedding into $\left(N, \leq_{\text {lex }}\right)$. Hence $N \in \boldsymbol{\Lambda}$.

Proposition 3.7 below will express that this example is typical for $\omega$-automatic $(2, \ell)$-partitions. But for its proof, we first need the following.

LEMMA 3.6. Let $L \subseteq \Sigma^{\omega}$ be regular and $\approx$ an automatic equivalence relation on $L$ with $|L / \approx| \geq \aleph_{1}$. Then there exist finite words $u_{i}, v_{i} \in \Sigma^{+}$such that $u_{i} v_{i}^{\omega} \in L$ and $\left[u_{i} v_{i}^{\omega}\right]_{\sim_{e}} \cap\left[u_{j} v_{j}^{\omega}\right]_{\approx}=\emptyset$ for all $0 \leq i<j$.

ProOf. Let $n \geq 0$ and suppose we have constructed pairs $\left(u_{i}, v_{i}\right)$ with the desired properties for $0 \leq i<n$. We want to find $\left(u_{n}, v_{n}\right)$. Suppose, for all $x \in L$, there exists $0 \leq i<n$ with $\left[u_{i} v_{i}^{\omega}\right]_{\sim_{e}} \cap[x]_{\approx} \neq \emptyset$. In other words, the countable set $\bigcup_{0<i<n}\left[u_{i} v_{i}^{\omega}\right]_{\sim_{e}}$ intersects every equivalence class of $\approx$. But this contradicts $|L /\{\approx\}|>\aleph_{0}$. Hence there exists an element $x \in L$ with $\left[u_{i} v_{i}^{\omega}\right]_{\sim_{e}} \cap[x]_{\approx}=\emptyset$ for all $i<n$, i.e., the formula

$$
\bigwedge_{0 \leq i<n} \neg \exists y: u_{i} v_{i}^{\omega} \sim_{e} y \approx x
$$

is satisfiable in the structure $\left(L,\left(u_{i} v_{i}^{\omega}\right), \sim_{e}, \approx\right)$. Since the relations $\sim_{e}, \approx$, and $\left\{u_{i} v_{i}^{\omega}\right\}$ for $0 \leq i<n$ are automatic, also the set of $\omega$-words $x$ satisfying this formula is automatic and therefore a nonempty regular $\omega$-language $[\mathbf{2}]$. Hence it contains some ultimately periodic $\omega$-word, i.e., there are $u_{n}, v_{n} \in \Sigma^{+}$as desired.

Let $u, v, w$ be some finite words. For $x \in\{0,1\}^{\omega}$, let $f_{v, w}(x)$ denote the $\omega$-word obtained from $x$ by replacing every occurrence of 0 by $v$ and every occurrence of 1 by $w$. Furthermore, $H_{u, v, w}=u f_{v, w}(N)$ where $N$ is the language from Example 3.5. 
Proposition 3.7. Let $P=\left(L, E_{0}, E_{1}, \ldots, E_{\ell}\right)$ be some $(2,1+\ell)$-partition with injective $\omega$-automatic presentation $(L, \mathrm{id})$ such that $\left\{(x, y) \mid\{x, y\} \in E_{0}\right\} \cup\{(x, x) \mid$ $x \in L\}$ is an equivalence relation (denoted $\approx$ ) on $L$ with at least $\aleph_{1}$ equivalence classes. Then there exist nonempty words $u, v$, and $w$ with $v$ and $w$ distinct, but of the same length, such that $H_{u, v, w}$ is $E_{i}$-homogeneous for some $1 \leq i \leq \ell$.

This proposition is stated in [11] where I explain how to obtain it from a proof in [1]. Here, we give a self-contained proof that follows and simplifies the ideas from $[1,11]$.

Proof. There are finite $\omega$-semigroups $S$ and $T$ and homomorphisms

$$
\varphi: \Sigma^{\infty} \rightarrow S \text { and } \psi:(\Sigma \times \Sigma)^{\infty} \rightarrow T
$$

such that $\varphi^{-1} \varphi(L)=L$ and

$$
\psi(x \otimes y)=\psi\left(x^{\prime} \otimes y^{\prime}\right) \Longrightarrow\left(\{x, y\} \in E_{i} \Longleftrightarrow\left\{x^{\prime}, y^{\prime}\right\} \in E_{i}\right)
$$

for all $0 \leq i \leq \ell$. Since the semigroups $S_{+}$and $T_{+}$are finite, there exists $\alpha \geq 1$ such that $x^{\alpha}$ is idempotent for all $x \in S_{+} \cup T_{+}$.

From Lemma 3.6, we find finite words $u_{i}, v_{i} \in \Sigma^{+}$such that $u_{i} v_{i}^{\omega} \in L$ and

$$
\left[u_{i} v_{i}^{\omega}\right]_{\sim_{e}} \cap\left[u_{j} v_{j}^{\omega}\right]_{\approx}=\emptyset
$$

for all $0 \leq i<j \leq\left|S_{+}\right| \cdot\left|T_{+}\right|$. Let $m$ be the maximal length of a word $u_{i}$ for $0 \leq i \leq\left|S_{+}\right| \cdot\left|T_{+}\right|$. By extending the word $u_{i}$ by the prefix of $v_{i}^{\omega}$ of length $m-\left|u_{i}\right|$ (and rotating the word $v_{i}$ accordingly), we can assume $\left|u_{i}\right|=\left|u_{j}\right|$ for all $0 \leq i<j \leq\left|S_{+}\right| \cdot\left|T_{+}\right|$without changing the words $u_{i} v_{i}^{\omega}$. Furthermore, we can replace $v_{i}$ by its

$$
\left(\alpha \cdot \prod_{\substack{0 \leq j \leq\left|S_{+}\right| \cdot\left|T_{+}\right| \\ i \neq j}}\left|v_{j}\right|\right)^{t h}
$$

power, i.e., we can assume that $\left|v_{i}\right|=\left|v_{j}\right|$ and that all the elements

$$
\varphi\left(v_{i}\right) \in S_{+} \text {and } \psi\left(v_{i} \otimes v_{j}\right) \in T_{+}
$$

are idempotent.

Now there are $0 \leq i<j \leq\left|S_{+}\right| \cdot\left|T_{+}\right|$with $\varphi\left(v_{i}\right)=\varphi\left(v_{j}\right)$ and $\psi\left(v_{i} \otimes v_{i}\right)=$ $\psi\left(v_{j} \otimes v_{j}\right)$. We may assume $i=0$ and $j=1$ and write, for simplicity,

$$
\begin{aligned}
& s=\varphi\left(v_{0}\right)=\varphi\left(v_{1}\right), \\
& t=\psi\left(v_{0} \otimes v_{0}\right)=\psi\left(v_{1} \otimes v_{1}\right), \\
& t_{01}=\psi\left(\left(v_{0} v_{0} v_{0} \otimes v_{0} v_{1} v_{0}\right)^{\alpha}\right) \text {, and } \quad t_{10}=\psi\left(\left(v_{0} v_{1} v_{0} \otimes v_{0} v_{0} v_{0}\right)^{\alpha}\right) \text {. }
\end{aligned}
$$

We will use the following equalities repeatedly:

$$
t_{01}=t_{01} \cdot t=t \cdot t_{01}=t_{01}^{2} \quad t_{10}=t_{10} \cdot t=t \cdot t_{10}=t_{10}^{2} \quad t=t^{2}
$$

Finally, for $X \subseteq \mathbb{N}$ we define a word $W_{X}$ as follows:

$$
W_{X}=u_{1} v_{1} \prod_{i \geq 0}\left\{\begin{array}{ll}
\left(v_{0} v_{0} v_{0}\right)^{\alpha} & \text { if } i \in X \\
\left(v_{0} v_{1} v_{0}\right)^{\alpha} & \text { if } i \notin X
\end{array}\right\}
$$

Note that, with $u=u_{1} v_{1}, v=\left(v_{0} v_{1} v_{0}\right)^{\alpha}$, and $w=\left(v_{0} v_{0} v_{0}\right)^{\alpha}$, we get $W_{X}=$ $u f_{v, w}\left(\chi_{X}\right)$ where $\chi_{X} \in\{0,1\}^{\omega}$ is the characteristic word of $X$.

We will now prove three claims concerning the words $W_{X}$.

Claim 1. $W_{X} \in L$ for all $X \subseteq \mathbb{N}$. 
Proof of Claim 1. We have $\varphi\left(W_{X}\right)=\varphi\left(u_{1}\right) \varphi\left(v_{1}\right) s^{\omega}=\varphi\left(u_{1} v_{1}^{\omega}\right)$ since $W_{X} \in$ $u_{1} v_{1}\left\{v_{0}, v_{1}\right\}^{\omega}$. Now $W_{X} \in L$ follows from $u_{1} v_{1}^{\omega} \in L$.

Claim 2. Let $X, Y \subseteq \mathbb{N}$ such that $X \backslash Y$ and $Y \backslash X$ are both infinite. Then

$$
\psi\left(W_{X} \otimes W_{Y}\right)=\psi\left(u_{1} \otimes u_{1}\right) \begin{cases}\left(t_{01} t_{10}\right)^{\omega} & \text { if } \min (X \triangle Y) \in X \\ \left(t_{10} t_{01}\right)^{\omega} & \text { if } \min (X \triangle Y) \in Y\end{cases}
$$

Proof of Claim 2.

$$
\begin{aligned}
\psi\left(W_{X} \otimes W_{Y}\right) & =\psi\left(u_{1} v_{1} \otimes u_{1} v_{1}\right) \prod_{0 \leq i}\left\{\begin{array}{ll}
\psi\left(v_{0} v_{0} v_{0} \otimes v_{0} v_{0} v_{0}\right)^{\alpha} & \text { if } i \in X \cap Y \\
\psi\left(v_{0} v_{0} v_{0} \otimes v_{0} v_{1} v_{0}\right)^{\alpha} & \text { if } i \in X \backslash Y \\
\psi\left(v_{0} v_{1} v_{0} \otimes v_{0} v_{0} v_{0}\right)^{\alpha} & \text { if } i \in Y \backslash X \\
\psi\left(v_{0} v_{1} v_{0} \otimes v_{0} v_{1} v_{0}\right)^{\alpha} & \text { otherwise }
\end{array}\right\} \\
& =\psi\left(u_{1} \otimes u_{1}\right) t \prod_{0 \leq i}\left\{\begin{array}{ll}
t_{01} & \text { if } i \in X \backslash Y \\
t_{10} & \text { if } i \in Y \backslash X \\
t^{3 \alpha}=t & \text { otherwise }
\end{array}\right\}
\end{aligned}
$$

Now the claim follows since there are infinitely many factors $t_{01}$ and $t_{10}$ (since $X \backslash Y$ and $Y \backslash X$ are both infinite), since these two factors are idempotent, and since multiplication of $t$ with $t_{10}$ or $t_{01}$ absorbes $t$.

q.e.d.

From Claim 2, we now derive that $H_{u, v, w}$ is homogeneous in the partition $P$ : Let $A, B \in\left[H_{u, v, w}\right]^{2}$. Then there exist sets $X, Y, X^{\prime}, Y^{\prime} \subseteq \mathbb{N}$ with $A=\left\{W_{X}, W_{Y}\right\}$ and $B=\left\{W_{X^{\prime}}, W_{Y^{\prime}}\right\}$. Furthermore, the differences $X \backslash Y, Y \backslash X, X^{\prime} \backslash Y^{\prime}$, and $Y^{\prime} \backslash X^{\prime}$ are all infinite. We assume $\min (X \triangle Y) \in X$ and $\min \left(X^{\prime} \triangle Y^{\prime}\right) \in X^{\prime}$. Then, by Claim 2, $\psi\left(W_{X} \otimes W_{Y}\right)=\psi\left(W_{X^{\prime}} \otimes W_{Y^{\prime}}\right)$ proving $A \in E_{i} \Longleftrightarrow B \in E_{i}$ for all $0 \leq i \leq \ell$. Hence $H_{u, v, w}$ is homogeneous. To show that it is not 0 -homogeneous, we use the following claim.

Claim 3. $\psi\left(W_{2 \mathbb{N}} \otimes W_{4 \mathbb{N}+2}\right)=\psi\left(u_{1} v_{1} v_{0}^{\omega} \otimes u_{1}\left(v_{1} v_{0}\right)^{\omega}\right)=\psi\left(u_{1}\left(v_{1} v_{0}\right)^{\omega} \otimes u_{1} v_{1}^{\omega}\right.$

Proof of Claim 3.

$$
\begin{aligned}
\psi\left(W_{2 \mathbb{N}} \otimes W_{4 \mathbb{N}+2}\right) & =\psi\left(u_{1} v_{1} \otimes u_{1} v_{1}\right) \\
& \cdot \prod_{0 \leq i}\left\{\begin{array}{ll}
\psi\left(v_{0} v_{0} v_{0} \otimes v_{0} v_{0} v_{0}\right)^{\alpha} & \text { if } i \in 4 \mathbb{N}+2 \\
\psi\left(v_{0} v_{0} v_{0} \otimes v_{0} v_{1} v_{0}\right)^{\alpha} & \text { if } i \in 4 \mathbb{N} \\
\psi\left(v_{0} v_{1} v_{0} \otimes v_{0} v_{1} v_{0}\right)^{\alpha} & \text { if } i \text { odd }
\end{array}\right\} \\
& =\psi\left(u_{1} \otimes u_{1}\right) t \prod_{0 \leq i}\left\{\begin{array}{ll}
t_{01} & \text { if } i \in 4 \mathbb{N} \\
t^{3 \alpha} & \text { otherwise }
\end{array}\right\} \\
& =\psi\left(u_{1} \otimes u_{1}\right) t_{01}^{\omega} \text { since } t t_{01}=t_{01} \\
\psi\left(u_{1} v_{1} v_{0}^{\omega}, u_{1}\left(v_{1} v_{0}\right)^{\omega}\right) & =\psi\left(u_{1} v_{1} \otimes u_{1} v_{1}\right) \psi\left(v_{0} v_{0} \otimes v_{0} v_{1}\right)^{\omega} \\
& =\psi\left(u_{1} \otimes u_{1}\right) t\left(t \psi\left(v_{0} \otimes v_{1}\right)\right)^{\omega} \\
& =\psi\left(u_{1} \otimes u_{1}\right)\left(t \psi\left(v_{0} \otimes v_{1}\right) t\right)^{\omega} \text { since } t^{2}=t \\
& =\psi\left(u_{1} \otimes u_{1}\right) t_{01}^{\omega} \operatorname{since}\left(t \psi\left(v_{0} \otimes v_{1}\right) t\right)^{\alpha}=t_{01} \\
\psi\left(u_{1}\left(v_{1} v_{0}\right)^{\omega} \otimes u_{1} v_{1}^{\omega}\right) & =\psi\left(u_{1} \otimes u_{1}\right)\left(t \psi\left(v_{0} \otimes v_{1}\right)\right)^{\omega} \\
& =\psi\left(u_{1} \otimes u_{1}\right)\left(t \psi\left(v_{0} \otimes v_{1}\right) t\right)^{\omega} \text { since } t^{2}=t \\
& =\psi\left(u_{1} \otimes u_{1}\right) t_{01}^{\omega} \operatorname{since}\left(t \psi\left(v_{0} \otimes v_{1}\right) t\right)^{\alpha}=t_{01}
\end{aligned}
$$


q.e.d.

Suppose $\left[H_{u, v, w}\right]^{2} \subseteq E_{0}$. Let $A \in\left[H_{u, v, w}\right]^{2}$, i.e., $A=\left\{W_{X}, W_{Y}\right\}$ for some $X, Y \subseteq \mathbb{N}$ with $X \backslash Y$ and $Y \backslash X$ infinite. We assume $\min (X \triangle Y) \in X$. From Claim 2, we obtain

$$
\psi\left(W_{X} \otimes W_{Y}\right)=\psi\left(W_{2 \mathbb{N}} \otimes W_{2 \mathbb{N}+1}\right)=\psi\left(W_{2 \mathbb{N}+1} \otimes W_{4 \mathbb{N}+2}\right) .
$$

Then $A \in E_{0}$ implies

$$
\left\{W_{2 \mathbb{N}}, W_{2 \mathbb{N}+1}\right\},\left\{W_{2 \mathbb{N}+1}, W_{4 \mathbb{N}+2}\right\} \in E_{0},
$$

in other words,

$$
W_{2 \mathbb{N}} \approx W_{2 \mathbb{N}+1} \approx W_{4 \mathbb{N}+2} .
$$

Since $\approx$ is an equivalence relation, this implies $W_{2 \mathbb{N}} \approx W_{4 \mathbb{N}+2}$. Now from Claim 3, we obtain $u_{1} v_{1} v_{0}^{\omega} \approx u_{1}\left(v_{1} v_{0}\right)^{\omega} \approx u_{1} v_{1}^{\omega}$. Since $u_{1} v_{1} v_{0}^{\omega} \sim_{e} u_{0} v_{0}^{\omega}$, this contradicts our choice of the words $u_{i}$ and $v_{i}$.

Theorem 3.8 (Kuske [11]). For all $\ell \geq 2$, we have

$$
\left(2^{\aleph_{0}}, \omega \mathrm{A}\right) \rightarrow\left(2^{\aleph_{0}}, \text { co-werCF } \cap \boldsymbol{\Lambda}\right)_{\ell}^{2} .
$$

Proof. Let $P^{\prime}=\left(V, E_{1}^{\prime}, \ldots, E_{\ell}^{\prime}\right)$ be some $(2, \ell)$ partition with $\omega$-automatic presentation $(L, h)$. To apply Prop. 3.7, consider the following $(2,1+\ell)$-partition $P=\left(L, E_{0}, \ldots, E_{\ell}\right)$ :

- The underlying set is the $\omega$-language $L$,

- $E_{0}$ consists of all sets $\{x, y\}$ with $h(x)=h(y)$ and $x \neq y$, and

- $E_{i}$ (for $\left.1 \leq i \leq \ell\right)$ consists of all sets $\{x, y\}$ with $\{h(x), h(y)\} \in E_{i}^{\prime}$.

Then $(L$, id $)$ is an injective $\omega$-automatic presentation of the $(2,1+\ell)$-partition $P$. By Prop. 3.7, there exists $1 \leq i \leq \ell$ and words $u, v$ and $w$ such that $v \neq w,|v|=|w|$, and such that $H_{u, v, w}$ is $E_{i}$-homogeneous for some $1 \leq i \leq \ell$. Since $\left(E_{0}, \ldots, E_{\ell}\right)$ is a partition of $[L]^{2}$, we have $\{x, y\} \notin E_{0}$ (and therefore $h(x) \neq h(y)$ ) for all $x, y \in H_{u, v, w}$ distinct. Hence $h$ is injective on $H_{u, v, w}$. Furthermore $\left[H_{u, v, w}\right]^{2} \subseteq E_{i}$ implies $\left[h\left(H_{u, v, w}\right)\right]^{2} \subseteq E_{i}^{\prime}$. Hence $h\left(H_{u, v, w}\right)$ is an $E_{i}^{\prime}$-homogeneous set of size $2^{\aleph_{0}}$. From $N \in$ co-werCF $\cap \boldsymbol{\Lambda}$, we get immediately $H_{u, v, w} \in \operatorname{co-\omega erCF} \cap \boldsymbol{\Lambda}$.

From Theorem 3.8, we now derive a necessary condition for a partial order of size $2^{\aleph_{0}}$ to be $\omega$-automatic. A partial order $(V$, exists a regular $\omega$-language $L$ and a surjection $h: L \rightarrow V$ such that the relations $R_{=}=\left\{(x, y) \in L^{2} \mid h(x)=h(y)\right\}$ and $R_{\sqsubseteq}=\left\{(x, y) \in L^{2} \mid h(x) \sqsubseteq h(y)\right\}$ are $\omega$-automatic.

COROLlaRY 3.9. If $(V, \sqsubseteq)$ is an $\omega$-automatic partial order with $|V| \geq \aleph_{1}$, then $(\mathbb{R}, \leq)$ or an antichain of size $2^{\aleph_{0}}$ embeds into $(V$,

Proof. Let $(V, \sqsubseteq)$ be a partial order, $L \subseteq \Sigma^{\omega}$ a regular $\omega$-language and $h$ : $L \rightarrow V$ a surjection such that $R_{=}$and $R_{\sqsubseteq}$ are $\omega$-automatic. Define an injective $\omega$-automatic $(2,4)$-partition $G=\left(L, E_{0}, E_{1}, E_{2}, E_{3}\right)$ :

- $E_{0}$ comprises all pairs $\{x, y\} \in[L]^{2}$ with $h(x)=h(y)$,

- $E_{1}$ comprises all pairs $\{x, y\} \in[L]^{2}$ with $h(x) \sqsubset h(y)$ and $x<_{\operatorname{lex}} y$,

- $E_{2}$ comprises all pairs $\{x, y\} \in[L]^{2}$ with $h(x) \sqsupset h(y)$ and $x<_{\operatorname{lex}} y$, and

- $E_{3}=[L]^{2} \backslash\left(E_{0} \cup E_{1} \cup E_{2}\right)$ comprises all pairs $\{x, y\} \in[L]^{2}$ such that $h(x)$ and $h(y)$ are incomparable. 
By Prop. 3.7, there exists $H \subseteq L 1$-, 2- or 3-homogeneous with $(\mathbb{R}, \leq) \hookrightarrow\left(H, \leq_{\text {lex }}\right)$. Since $[H]^{2} \subseteq E_{1} \cup E_{2} \cup E_{3}$ and since $G$ is a partition of $L$, the mapping $h$ acts injectively on $H$. If $[H]^{2} \subseteq E_{1}$ (the case $[H]^{2} \subseteq E_{2}$ is symmetrical) then $(\mathbb{R}, \leq) \hookrightarrow$ $\left(H, \leq_{\text {lex }}\right) \cong(h(H), \sqsubseteq)$. If $[H]^{2} \subseteq E_{3}$, then $h(H)$ is an antichain of size $2^{\aleph_{0}}$.

A linear order $(L, \sqsubseteq)$ is scattered if $(\mathbb{Q}, \leq)$ cannot be embedded into $(L, \sqsubseteq)$. Automatic partial orders are defined similarly to $\omega$-automatic partial orders with the help of finite automata instead of Büchi-automata.

Corollary 3.10. Any scattered $\omega$-automatic linear order $(V, \sqsubseteq)$ is countable. Hence,

- a scattered linear order is $\omega$-automatic if and only if it is automatic, and

- an ordinal $\alpha$ is $\omega$-automatic if and only if $\alpha<\omega^{\omega}$.

Proof. If $(V, \sqsubseteq)$ is not countable, then it embeds $(\mathbb{R}, \leq)$ by the previous corollary and therefore in particular $(\mathbb{Q}, \leq)$. The remaining two claims follow immediately from [1] ("countable $\omega$-automatic structures are automatic") and [4] ("an ordinal is automatic iff it is properly smaller than $\left.\omega^{\omega "}\right)$, resp.

3.2.3. Sierpinski's theorem for $\omega$-automatic partitions. Compare Theorem 3.8 to Sierpiński's theorem 3.1 and the theorem of Erdös and Rado: if an edge-labeled graph is $\omega$-automatic, already $2^{\aleph_{0}}$ nodes guarantee the existence of a homogeneous set of size $2^{\aleph_{0}}$. Compared to Rubin's theorem 2.12, there are two shortcomings: We would like to extend the result to $(k, \ell)$-partitions for $k>2$, and we would like to find $\omega$-regular homogeneous sets (and not only complements of context-free ones). The following two examples prove that this is not possible, i.e., that Theorem 3.8 is best possible.

Example 3.11. Let $\Sigma=\{0,1\}, L=\{0,1\}^{\omega}$. For $H \subseteq L$, we write $\bigwedge H \in \Sigma^{\infty}$ for the longest common prefix of all $\omega$-words in $H, \bigwedge\{x, y\}$ is also written $x \wedge y$. By $\leq_{\text {lex }}$, we denote the lexicographic order on the set $\Sigma^{\omega}$ (with some, implicitly assumed linear order on the letters from $\Sigma$ ). Then let $E_{1}$ consist of all 3 -sets $\{x, y, z\} \in[L]^{3}$ with $x<_{\text {lex }} y<_{\text {lex }} z$ and $x \wedge y<_{\text {pref }} y \wedge z ; E_{2}$ is the complement of $E_{1}$. This finishes the construction of the $(3,2)$-partition $\left(L, E_{1}, E_{2}\right)$ of size $2^{\aleph_{0}}$ with injective $\omega$-automatic presentation $(L$, id $)$.

Note that $1^{*} 0^{\omega}$ is a countable $E_{1}$-homogeneous set and that $0^{*} 1^{\omega}$ is a countable $E_{2}$-homogeneous set. But there is no uncountable homogeneous set: First suppose $H \subseteq L$ is infinite and $x \wedge y<_{\text {pref }} y \wedge z$ for all $x<_{\operatorname{lex}} y<_{\operatorname{lex}} z$ from $H$. Let $u \in \Sigma^{*}$ such that $H \cap u 0 \Sigma^{\omega}$ and $H \cap u 1 \Sigma^{\omega}$ are both nonempty and let $x, y \in H \cap u 0 \Sigma^{\omega}$ with $x \leq_{\text {lex }} y$ and $z \in H \cap u 1 \Sigma^{\omega}$. Then $x \wedge y>_{\text {pref }} u=y \wedge z$ and therefore $x=y$ (for otherwise, we would have $x<_{\text {lex }} y<_{\text {lex }} z$ in $H$ with $x \wedge y>_{\text {pref }} y \wedge z$ ). Hence we showed $\left|H \cap u 0 \Sigma^{\omega}\right|=1$. Let $u_{0}=\bigwedge H$ and $H_{1}=H \cap u_{0} 1 \Sigma^{\omega}$. Since $H \cap u_{0} 0 \Sigma^{\omega}$ is finite, the set $H_{1}$ is infinite. We proceed by induction: $u_{n}=\bigwedge H_{n}$ and $H_{n+1}=H_{n} \cap u_{n} 1 \Sigma^{\omega}$ satisfying $\left|H_{n} \cap u_{n} 0 \Sigma^{\omega}\right|=1$. Then $u_{0}<_{\text {pref }} u_{0} 1 \leq_{\text {pref }}$ $u_{1}<_{\text {pref }} u_{1} 1 \leq_{\text {pref }} u_{2} \cdots$ with

$$
H=\bigcup_{n \geq 0}\left(H \cap u_{n} 0 \Sigma^{\omega}\right) \cup \bigcap_{n \geq 0}\left(H \cap u_{n} 1 \Sigma^{\omega}\right) .
$$

Then any of the sets $H \cap u_{n} 0 \Sigma^{\omega}=H_{n} \cap u_{n} 0 \Sigma^{\omega}$ and $\bigcap\left(H \cap u_{n} 1 \Sigma^{\omega}\right)$ is a singleton, proving that $H$ is countable. Thus, there cannot be an uncountable $E_{1^{-}}$ homogeneous set. 
So let $H \subseteq L$ be infinite with $x \wedge y \geq_{\text {pref }} y \wedge z$ for all $x<_{\text {lex }} y<_{\text {lex }} z$. Since we have only two letters, we get $x \wedge y>_{\text {pref }} y \wedge z$ for all $x<_{\text {lex }} y<_{\text {lex }} z$ which allows to argue symmetrically to the above. Thus, indeed, there is no uncountable homogeneous set in $L$. This proves $\left(2^{\aleph_{0}}, \omega \mathrm{iA}\right) \not \rightarrow\left(\aleph_{1}, \omega \mathrm{LANG}\right)_{2}^{3}$; using the trick from the end of the proof of Sierpiński's theorem 3.1 (where $x_{1}<x_{2}$ is replace by $\left.x_{1}<_{\operatorname{lex}} x_{2}\right)$, we get $\left(2^{\aleph_{0}}, \omega \mathrm{iA}\right) \not \rightarrow\left(\aleph_{1}, \omega \mathrm{LANG}\right)_{\ell}^{k}$ for all $k \geq 3$ and $\ell \geq 2$.

EXAMPLE 3.12. Let $L$ denote the regular $\omega$-language $\left(1^{+} 0^{+}\right)^{\omega}$. Recall that the $\omega$-words $x$ and $y$ are ultimately equal, briefly $x \sim_{e} y$, if there exist finite words $u$ and $v$ of the same length and an $\omega$-word $z$ with $x=u z$ and $y=v z$ (i.e., from some point on, the two $\omega$-words coincide). Let $E_{1} \subseteq[L]^{2}$ consists of all 2-sets $\{x, y\} \subseteq L$ such that $\operatorname{supp}(x) \cap \operatorname{supp}(y)$ is finite or $x \sim_{e} y$. The set $E_{2}$ is the complement of $E_{1}$ in $[L]^{2}$. This completes the construction of the $(2,2)$-partition $G=\left(L, E_{1}, E_{2}\right)$. Note that $\left(L, \operatorname{id}_{L}\right)$ is an injective $\omega$-automatic presentation of $G$.

Now let $n \in \mathbb{N}$ and $U_{i}, V_{i} \subseteq\{0,1\}^{+}$be languages, set

$$
H=\bigcup_{1 \leq i \leq n} U_{i} V_{i}^{\omega}
$$

and assume that $H$ has size $2^{\aleph_{0}}$. We show that $H$ is not homogeneous: Since $H$ is infinite, there are $1 \leq i \leq n$ and $x, y \in U_{i} V_{i}^{\omega}$ distinct with $x \sim_{e} y$ and therefore $\{x, y\} \in E_{1}$.

Since $|H|>\aleph_{0}$, there is $1 \leq i \leq n$ with $\left|U_{i} V_{i}^{\omega}\right|>\aleph_{0}$; we set $U=U_{i}$ and $V=V_{i}$. Let $u \in U$ be arbitrary (such a word exists since $U V^{\omega} \neq \emptyset$ ). From $|U| \leq \aleph_{0}$, we obtain $\left|V^{\omega}\right|>\aleph_{0}$. Hence there are $v_{1}, v_{2} \in V^{+}$distinct with $\left|v_{1}\right|=\left|v_{2}\right|$. Since $u v_{1}^{\omega} \in H$ and each element of $H$ contains infinitely many occurrences of 1 , the word $v_{1}$ belongs to $\{0,1\}^{*} 10^{*}$. Consider the $\omega$-words $x^{\prime}=u\left(v_{1} v_{2}\right)^{\omega}$ and $y^{\prime}=u\left(v_{1} v_{1}\right)^{\omega}$ from $U V^{\omega} \subseteq H$. Then $x^{\prime} \chi_{e} y^{\prime}$ since $v_{1} \neq v_{2}$ and $\left|v_{1}\right|=\left|v_{2}\right|$. At the same time, $\operatorname{supp}\left(x^{\prime}\right) \cap \operatorname{supp}\left(y^{\prime}\right)$ is infinite since $v_{1}$ contains an occurrence of 1 . Hence $\left\{x^{\prime}, y^{\prime}\right\} \in E_{2}$.

Thus, we found $\omega$-words $x, y, x^{\prime}, y^{\prime} \in H$ with $\{x, y\} \in E_{1}$ and $\left\{x^{\prime}, y^{\prime}\right\} \notin E_{1}$ proving that $H$ is not homogeneous.

Since all context-free and regular $\omega$-language are of the form $(3.1)$ (see [18]), this proves $\left(2^{\aleph_{0}}, \omega \mathrm{iA}\right) \not \rightarrow\left(2^{\aleph_{0}}, \omega \mathrm{CF}\right)_{\ell}^{k}$ and $\left(2^{\aleph_{0}}, \omega \mathrm{iA}\right) \not \rightarrow\left(2^{\aleph_{0}}, \omega \mathrm{REG}\right)_{\ell}^{k}$ for all $k, \ell \geq 2$.

\section{References}

[1] V. Bárány, Ł. Kaiser, and S. Rubin, Cardinality and counting quantifiers on omega-automatic structures, STACS'08, IFIB Schloss Dagstuhl, 2008, pp. 385-396.

[2] A. Blumensath, Automatic structures, Tech. report, RWTH Aachen, 1999.

[3] A. Blumensath and E. Grädel, Finite presentations of infinite structures: Automata and interpretations, Theory of Computing Systems 37 (2004), no. 6, 641-674.

[4] Ch. Delhommé, Automaticité des ordinaux et des graphes homogènes, C. R. Acad. Sci. Paris, Ser. I 339 (2004), 5-10.

[5] P. Erdős and R. Rado, A problem on ordered sets, Journal of the LMS 28 (1953), 426-438.

[6] A partition calculus in set theory, Bull. AMS 62 (1956), 427-489.

[7] C.G. Jockusch, Ramsey's theorem and recursion theory, Journal of Symbolic Logic 37 (1972), $268-280$.

[8] C.G. Jockusch and R.I. Soare, $\Pi_{1}^{0}$-classes an degrees of theories, Trans. Am. Math. Soc. 173 (1972), 33-56.

[9] B. Khoussainov and A. Nerode, Automatic presentations of structures, Logic and Computational Complexity, Lecture Notes in Comp. Science vol. 960, Springer, 1995, pp. 367-392.

[10] B. Khoussainov, S. Rubin, and F. Stephan, On automatic partial orders, LICS'03, IEEE Computer Society Press, 2003, pp. 168-177. 
[11] D. Kuske, Is Ramsey's theorem w-automatic?, STACS 2010, Leibniz International Proceedings in Informatics (LIPIcs) vol. 5, Schloss Dagstuhl-Leibniz-Zentrum für Informatik, 2010, pp. $537-548$.

[12] D. Kuske and M. Lohrey, Some natural problems in automatic graphs, Journal of Symbolic Logic 75 (2010), no. 2, 678-710.

[13] D. Perrin and J.-E. Pin, Infinite words, Pure and Applied Mathematics vol. 141, Elsevier, 2004.

[14] F.P. Ramsey, On a problem of formal logic, Proc. London Math. Soc. 30 (1930), 264-286.

[15] S. Rubin, Automata presenting structures: A survey of the finite string case, Bulletin of Symbolic Logic 14 (2008), 169-209.

[16] J.R. Shoenfield, On degrees of unsolvability, Annals of Mathematics 69 (1959), no. 3, $644-653$.

[17] W. Sierpiński, Sur un problème de la thèorie des relations, Ann. Scuola Norm. Sup. Pisa 2 (1933), no. 2, 285-287.

[18] L. Staiger, $\omega$-languages, Handbook of Formal Languages Vol. 3 (G. Rozenberg and A. Salomaa, eds.), Springer, 1997, pp. 339-387.

Institut Für Theoretische Informatik, TU Ilmenau, PF 100565, D-98684 Ilmenau, Germany

E-mail address: dietrich.kuske@tu-ilmenau.de 